\title{
Practical Synthesis of 2-Iodosobenzoic Acid (IBA) without Contamination by Hazardous 2-Iodoxybenzoic Acid (IBX) under Mild Conditions
}

\author{
Hideyasu China ${ }^{1,2, *}$, Nami Kageyama ${ }^{2}$, Hotaka Yatabe ${ }^{2}$, Naoko Takenaga ${ }^{3}$ (D) and Toshifumi Dohi ${ }^{2, *(D)}$ \\ 1 Department of Medical Bioscience, Nagahama Institute of Bio-Science and Technology, 1266, \\ Tamuracho Nagahama-shi, Shiga 526-0829, Japan \\ 2 College of Pharmaceutical Sciences, Ritsumeikan University, 1-1-1 Nojihigashi, Kusatsu, Shiga 525-8577, \\ Japan; ph0093ei@ed.ritsumei.ac.jp (N.K.); ph0090ir@ed.ritsumei.ac.jp (H.Y.) \\ 3 Faculty of Pharmacy, Meijo University, 150 Yagotoyama, Tempaku-ku, Nagoya 468-8503, Japan; \\ ntakenag@meijo-u.ac.jp \\ * Correspondence: h_china@nagahama-i-bio.ac.jp (H.C.); td1203@ph.ritsumei.ac.jp (T.D.)
}

check for updates

Citation: China, H.; Kageyama, N.; Yatabe, H.; Takenaga, N.; Dohi, T.

Practical Synthesis of

2-Iodosobenzoic Acid (IBA) without Contamination by Hazardous 2-Iodoxybenzoic Acid (IBX) under Mild Conditions. Molecules 2021, 26, 1897. https://doi.org/10.3390/ molecules 26071897

Academic Editor: Maged Henary

Received: 28 February 2021

Accepted: 24 March 2021

Published: 27 March 2021

Publisher's Note: MDPI stays neutral with regard to jurisdictional claims in published maps and institutional affiliations.

Copyright: (C) 2021 by the authors. Licensee MDPI, Basel, Switzerland. This article is an open access article distributed under the terms and conditions of the Creative Commons Attribution (CC BY) license (https:// creativecommons.org/licenses/by/ $4.0 /)$.

\begin{abstract}
We report a convenient and practical method for the preparation of nonexplosive cyclic hypervalent iodine(III) oxidants as efficient organocatalysts and reagents for various reactions using Oxone ${ }^{\circledR}$ in aqueous solution under mild conditions at room temperature. The thus obtained 2-iodosobenzoic acids (IBAs) could be used as precursors of other cyclic organoiodine(III) derivatives by the solvolytic derivatization of the hydroxy group under mild conditions of $80{ }^{\circ} \mathrm{C}$ or lower temperature. These sequential procedures are highly reliable to selectively afford cyclic hypervalent iodine compounds in excellent yields without contamination by hazardous pentavalent iodine(III) compound.
\end{abstract}

Keywords: cyclic organoiodine(III) compounds; Oxone ${ }^{\circledR}$; water, solvolytic functionalization, mild condition, metal-free, 2-iodosobenzoic acid

\section{Introduction}

Cyclic hypervalent iodine reagents, such as 2-iodosobenzoic acid (IBA) and 2-iodoxybenzoic acid (IBX) are nonmetallic green oxidants with excellent recyclability [1-3]. IBA and IBX can be regenerated from 2-iodobenzoic acid (2-IB) without requiring an external ligand except for water in this reoxidation step. This is because the carboxy group adjacent to the iodine atom serves as an endogenous ligand. Recently, IBA, a representative trivalent cyclic hypervalent iodine oxidant, has been used as a catalyst and reagent in various reactions, i.e., decarboxylative alkynylation [4,5], decarboxylative acylarylation [6], oxyalkenylation [7], oxyarylation [8], oxidative C-H arylation [9], C-H hydroxylation [10], C-H oxidation [11,12], ring-opening hydrazination [13], and asymmetric intramolecular $\alpha$-cyclopropanation [14]. IBA derivatives containing OAc [15-28], OMe [29-32], OTs [33-35], OTf [36,37], Cl [38-42], F [43-45], CN [46], N 3 [47-54], $\mathrm{CF}_{3}[55,56], \mathrm{OCOCF}_{3}$ [57], alkynyl [58-62] ligands instead of the hydroxy group have also found application in various reactions (Figure 1).

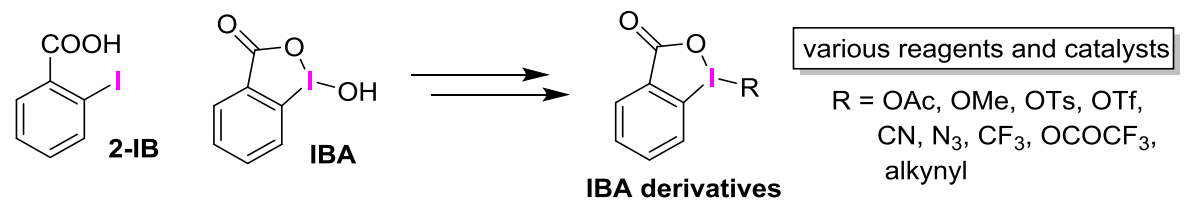

Figure 1. 2-Iodosobenzoic acid (IBA) and its precursor 2-iodobenzoic acid (2-IB).

Although IBAs can be prepared from 2-IBs by existing methods (Figure 2) [63-66], the development of a safer and more efficient method for their synthesis is highly desirable. 
As shown in Figure 2, IBAs can be further oxidized to pentavalent cyclic hypervalent IBXs [67], which need to be prevented for the preparation of IBAs [68-70], mainly due to the explosive nature of IBXs on heating and impact, while IBXs are useful in some small-scale reactions [71-76]. Thus, contamination by IBX in the IBA products should be avoided for long-term safe storage or large-scale use.

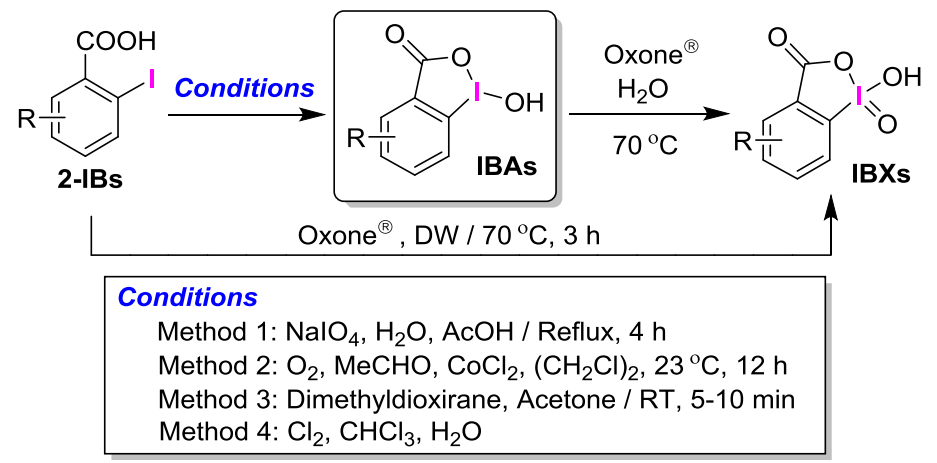

Figure 2. Synthesis of 2-iodosobenzoic acids (IBAs) and 2-iodoxybenzoic acids (IBXs) from 2iodobenzoic acids (2-IBs).

In recent decades, many reactions using Oxone ${ }^{\circledR}$, which is an inexpensive and commercially available stable triple salt $\left(2 \mathrm{KHSO}_{5} / \mathrm{KHSO}_{4} / \mathrm{K}_{2} \mathrm{SO}_{4}\right)$, have been developed for practical synthetic purposes [77]. In particular, the use of Oxone ${ }^{\circledR}$ as a re-oxidant for pentavalent hypervalent iodine reagents is drawing attention for catalytic oxidation reactions [78-92]. The reaction systems for alcohol oxidations [78-84] involving in situ generated active hypervalent iodine $(\mathrm{V})$ species are optimized on the basis of the preparative conditions of IBX from 2-IB at $70{ }^{\circ} \mathrm{C}$ [93]. Meanwhile, oxidative lactonizations from modified 2-IBs using Oxone ${ }^{\circledR}$ occur at room temperature [85-87]. In this context, the generation of nonexplosive trivalent cyclic hypervalent iodines, i.e., IBA and its analogs, using Oxone ${ }^{\circledR}$ can be expected to provide a convenient and safe synthetic procedure; however, to best of our knowledge, the selective preparation of IBAs using Oxone ${ }^{\circledR}$ has not been reported so far.

Recently, we reported that IBAs generated in a reaction system containing 2-IB and Oxone ${ }^{\circledR}$ play a catalytic role in the selective oxidation of alkoxybenzenes to $p$-quinones [94]. This resulted in the development of the practical method herein reported for the selective preparation of IBAs under mild conditions.

\section{Results and Discussion}

\subsection{Selective Synthesis of IBA and Its Analogs}

We started our investigation on the selective preparation of IBAs by evaluating the solvent effects on the oxidation of 2-IB 1a using 1.0 equivalent of Oxone ${ }^{\circledR}$ to obtain IBA $2 \mathbf{a}$ as a representative compound, and the results are summarized in Figure 3. First, the reaction in water led to the successful production of IBA $\mathbf{2 a}$ in $82 \%$ yield (Figure 3, entry 2), whereas IBA 2a was not produced in organic solvent in the absence of water (no reaction because Oxone ${ }^{\circledR}$ was not dissolved) (see entry 1 ). This result indicates that water plays an essential role in the formation of IBA. Therefore, we assumed that an aqueous system similar to the selective formation of $p$-quinone from alkoxybenzenes catalyzed by 2-IB 1a with Oxone ${ }^{\circledR}$ [94] could be suitable for the present reaction. We then investigated in detail the effect of a series of organic solvents on the aqueous preparation of IBA 2a using Oxone ${ }^{\circledR}$. 


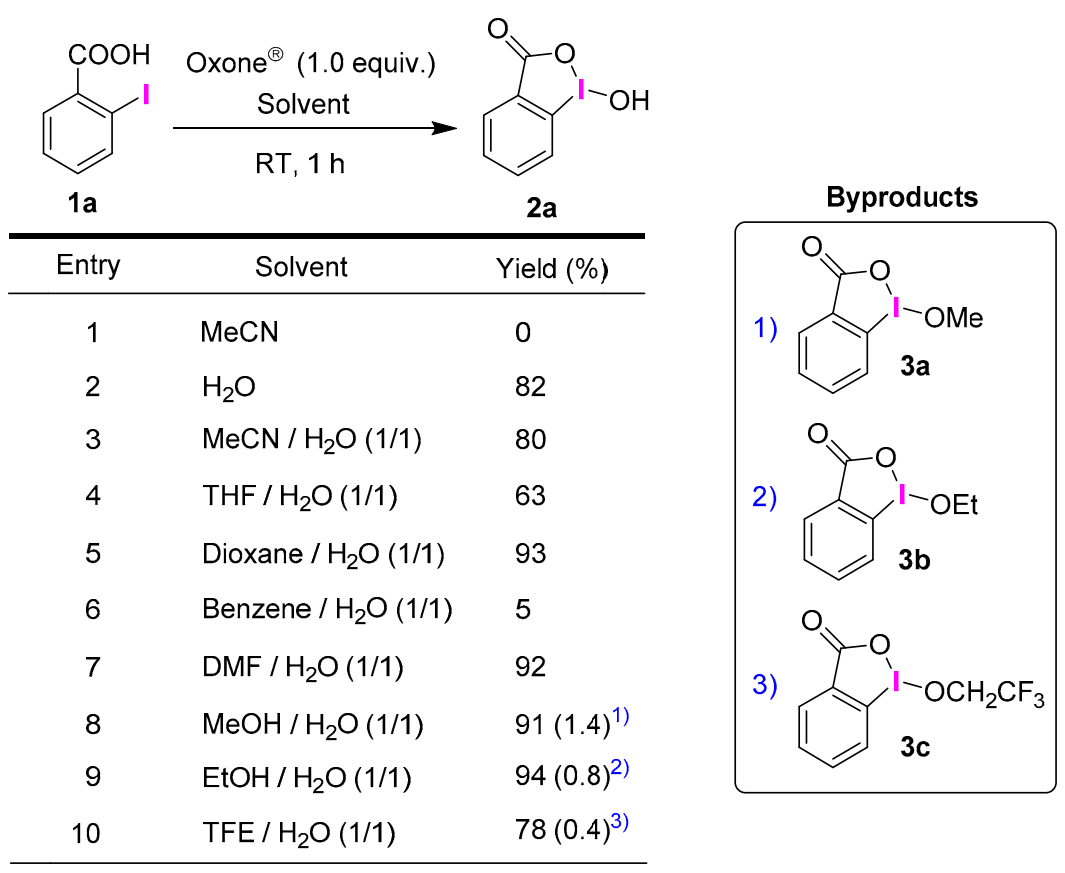

Figure 3. Solvent effect on the selective synthesis of IBA 2a from 2-IB 1a using Oxone ${ }^{\circledR}$.

Various water-miscible organic solvents were investigated to dissolve 2-IB 1a in this reaction. The preparation of IBA 2a using acetonitrile $(\mathrm{MeCN})$ in aqueous condition (Figure 3, entry 3) was similar to that performed in the absence of organic solvents (Figure 3, entry 2). Tetrahydrofuran (THF), dioxane, benzene and $N, N$-dimethylformamide (DMF) were also examined, finding that the use of highly polar dioxane and DMF led to excellent yields of IBA 2a (Figure 3, entries 5 and 7), whereas benzene, the least polar solvent among these aprotic solvents, significantly reduced the yield of the desired product (Figure 3, entry 6). The reason for this very low yield IBA formation was interpreted as being due to that benzene forms a two-phase system and interferes with the dissolution of 2-IB into water. Protic solvents such as $\mathrm{MeOH}, \mathrm{EtOH}$ and 2,2,2-trifuoroethanol (TFE) gave IBA 2a in high yields; however, they also worked as a ligand for IBA, causing the formation of very small amounts of ligand-exchanged byproducts 3a-c (Figure 3, entries 8-10). The white solid IBA 2a obtained after the water and acetone washings did not contain any other byproducts. Although this result indicated that protic organic solvents were not suitable for the selective preparation of IBAs, it also revealed that the IBA hydroxyl group could undergo substitution reactions under mild conditions (vide infra). The yields indicated in Figure 3 are almost equal to the conversion of 2-IB 1a.

Next, we investigated the substrate scope for the synthesis of IBAs using Oxone ${ }^{\circledR}$ under aqueous conditions with $\mathrm{MeCN}$, and the results are shown in Figure 4. MeCN was used as a component of the solvent to dissolve substrates 1 . By oxidation of 5-substituted 2IBs, IBAs 2b-d containing fluoro-, chloro-, and bromo-substituents were smoothly obtained in excellent yields from the corresponding halo-substituted 2-IBs $\mathbf{1 b}-\mathbf{d}$. From 2-IBs 1e-j with electron-donating groups such as methyl-, methoxy-, and acethoxy-substituents (1e-g) and electron-withdrawing groups such as trifluoromethyl-, nitro- and cyano-substituents $(\mathbf{1} \mathbf{h}-\mathbf{j})$, the desired IBAs $\mathbf{2} \mathbf{e}-\mathbf{j}$ were also produced in good yields. However, 2-IB bearing a hydroxy-substituent $\mathbf{1} \mathbf{k}$ afforded the desired product $\mathbf{2 k}$ in a moderate yield under the same conditions. In the oxidation of 4-substituted 2-IBs, fluoro-, chloro-, bromo-, trifluoromethyl-, and carboxy-substituted IBAs $\mathbf{2 1 - p}$ were obtained in excellent yields from the corresponding 2-IBs 11-p. In addition, the oxidized products of 4,5-disubstituted 2-IBs containing difluoro-substituents $\mathbf{2 q}$ and dimethoxy-substituents $\mathbf{2 r}$ were obtained in high yields. Meanwhile, with regard to 3-substituted 2-IBs, the reaction of methyl-substituted 1t with a slight excess of Oxone ${ }^{\circledR}$ afforded the expected IBA $2 t$ in a good yield, whereas 
the yield of bromo-substituted IBA $2 \mathbf{s}$ was lower even at the elevated temperature and in the presence of a large excess of Oxone ${ }^{\circledR}$. Steric effects are probably important in the formation of the cyclic $\lambda^{3}$-iodanes. Indeed, the presence of a substituent at the ortho position of the iodine atom (3-position) interfered in the synthesis of the corresponding product for 3-bromo-substituted 2-IB 1s. In the case of 6-substituted 2-IBs, fluoro-substituted IBA $2 \mathbf{u}$ and methyl-substituted $2 \mathbf{v}$ were obtained in good yields. Finally, the reaction of 3-iodonaphthalene-2-carboxylic acid 3 under the present conditions led to the expected tricyclic hypervalent iodine compound 4 in an excellent yield (Scheme 1).

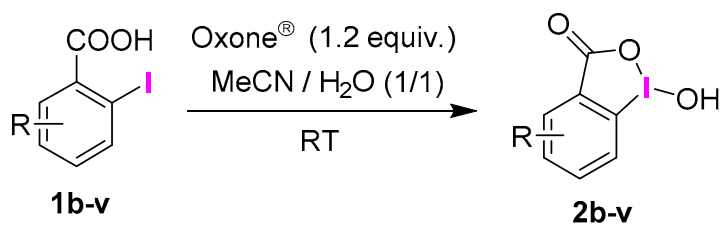<smiles>[R]c1ccc2ooc(=O)c2c1</smiles>

$(\mathrm{R}=\mathrm{F} ; \mathbf{2 b}): 95 \%(2 \mathrm{~h})$

$(\mathrm{R}=\mathrm{Cl} ; \mathbf{2 c}): 95 \%(3 \mathrm{~h})$

$(\mathrm{R}=\mathrm{Br} ; \mathbf{2 d}): 90 \%(12 \mathrm{~h})$

$(\mathrm{R}=\mathrm{Me} ; \mathbf{2 e}): 84 \%(15 \mathrm{~h})$

$(\mathrm{R}=\mathrm{OMe} ; \mathbf{2 f}): 77 \%(3 \mathrm{~h})$

$(\mathrm{R}=\mathrm{OAc} ; \mathbf{2 g}): 82 \%(2 \mathrm{~h})$

$\left(\mathrm{R}=\mathrm{CF}_{3} ; \mathbf{2 h}\right): 82 \%(20 \mathrm{~h})$

$\left(\mathrm{R}=\mathrm{NO}_{2} ; \mathbf{2 i}\right): 73 \%(24 \mathrm{~h})$

$(\mathrm{R}=\mathrm{CN} ; \mathbf{2 j}): 84 \%(15 \mathrm{~h})$

$(\mathrm{R}=\mathrm{OH} ; \mathbf{2 k}): 60 \%(3 \mathrm{~h})$<smiles>[R]c1ccc2c(=O)ooc2c1</smiles>

( $R=F ; 2 I): 94 \%(15 h)$

$(\mathrm{R}=\mathrm{Cl} ; \mathbf{2 m}): 85 \%(3 \mathrm{~h})$

$(\mathrm{R}=\mathrm{Br} ; \mathbf{2 n}): 91 \%(24 \mathrm{~h})$

$\left(\mathrm{R}=\mathrm{CF}_{3} ; 2 \mathrm{o}\right): 65 \%(20 \mathrm{~h})$

$(\mathrm{R}=\mathrm{COOH} ; 2 \mathrm{p}): 94 \%(18 \mathrm{~h})$<smiles>[R]c1cc2c(cc1[R])I(O)OC2=O</smiles>

$(R=F ; 2 q): 91 \%(15 h)$

(R = OMe; $2 r): 72 \%(5 \mathrm{~h})$<smiles>[R]c1cccc2c(=O)ooc12</smiles>

$(R=B r ; 2 s): 40 \%(24 h)^{a)}$

$14 \%(15 h)^{b)}$

$\left.(R=M e ; 2 t): 90 \%(15 h)^{b}\right)$<smiles>[R]c1cccc2c(=O)ooc12</smiles>

$(\mathrm{R}=\mathrm{F} ; \mathbf{2 u}): 88 \%(15 \mathrm{~h})$

$(\mathrm{R}=\mathrm{Me} ; \mathbf{2 v}): 74 \%(20 \mathrm{~h})$

Figure 4. Substrate scope of the selective preparation of IBAs $\mathbf{2 b}-\mathbf{v}$ using Oxone ${ }^{\circledR}$ under aqueous conditions. (a) This reaction was performed at $30{ }^{\circ} \mathrm{C}$ in the presence of 1.2 equivalents of Oxone ${ }^{\circledR}$. (b) Performed using 5 equivalents of Oxone ${ }^{\circledR}$ at $40^{\circ} \mathrm{C}$.

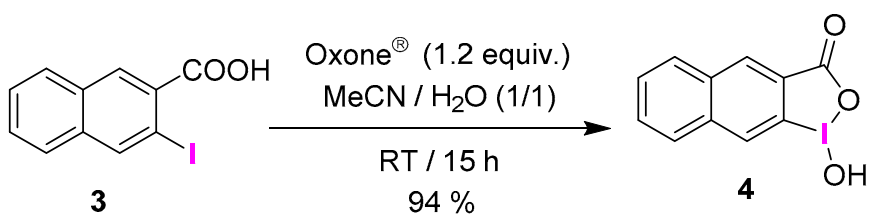

Scheme 1. Synthesis of tricyclic hypervalent iodine compound 4.

\subsection{IBAs Synthesis Using Ferric Effect}

As mentioned in Section 2.1, our present method can selectively afford trivalent cyclic hypervalent iodine IBA at room temperature without contamination by pentavalent iodine byproduct. The mild conditions used contributed favorably to this product selectivity. Interestingly, we further found that iron ion in tap water (TW), which contained iron ion (5.8 $\mu \mathrm{M}$ or less), contribute to the IBA formation, whereas calcium and magnesium ions as main minerals in TW do not affect the selectivity. Indeed, IBA $2 \mathbf{a}$ was selectively produced from 2-IB 1a by heating even at $100{ }^{\circ} \mathrm{C}$ in DW containing $5 \mathrm{~mol} \% \mathrm{FeCl}_{3}$ (Scheme 2, left). On the other hand, IBX was instead formed as a main product in the absence of $\mathrm{FeCl}_{3}$ in 
deionized water (DW) [93]. Other ferric salts such as $\mathrm{Fe}\left(\mathrm{NO}_{3}\right), \mathrm{Fe}(\mathrm{OTf})_{3}$, and $\mathrm{FeSO}_{4}$ had similar effects. In addition, it was found that pentavalent IBX 5 was converted to IBA $2 \mathbf{a}$ in the presence of a catalytic amount of $\mathrm{FeCl}_{3}$ at $100{ }^{\circ} \mathrm{C}$ (Scheme 2, right), while the formation of unidentified high- and low-polar decomposition products were detected in the water and the acetone washing solution, respectively. Here, 2-IB 1a was not produced. These results would indicate that overoxidation of IBA 2a to pentavalent IBX 5 was strongly prevented by ferric salts. Thus, the effect of the metal ion in the decomposition of hazardous IBX 5 is also a significant key factor to ensure the safety for our trivalent cyclic hypervalent iodine synthesis under heating conditions.<smiles>O=C(O)c1ccccc1I</smiles>

$1 \mathrm{a}$

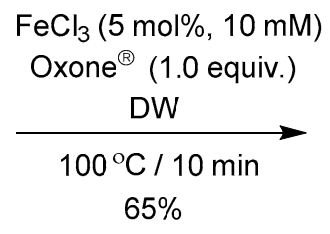
$65 \%$<smiles>O=c1ooc2ccccc12</smiles>

$2 a$<smiles>O=C1OI(=O)(O)c2ccccc21</smiles>

IBX (5)

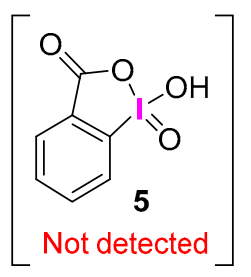

Scheme 2. Effect of a ferric salt on the selective synthesis of IBA 2a.

The reaction time in the synthesis of IBAs was significantly shortened by heating. In the investigation of the heating conditions for the synthesis of IBA $2 \mathbf{a}$ in $0.2 \mathrm{M} 2$-IB 1a in the presence of $2.5 \mathrm{~mol}^{\%} \mathrm{FeCl}_{3}$ for $10 \mathrm{~min}$, the required amount of Oxone ${ }^{\circledR}$ and the reaction temperature were thus optimized to $60{ }^{\circ} \mathrm{C}$ (Figure 5a) and 1.0 equivalent (Figure $5 b$ ), respectively. The yield of IBA $2 a$ was very sensitive to the reaction time, which dropped from $83 \%$ for $10 \mathrm{~min}$ to $70 \%$ for $1 \mathrm{~h}$. IBA may be decomposed to small molecules in the presence of excess Oxone ${ }^{\circledR}$; it has been reported that Oxone ${ }^{\circledR}$ causes oxidative cleavage of the aromatic ring [95]. Without Oxone ${ }^{\circledR}$, we also confirmed that IBA 2a was hardly decomposed under the conditions of Scheme 2 in the presence of 1.0 equivalent of $\mathrm{H}_{2} \mathrm{SO}_{4}$ and $10 \mathrm{~mol} \% \mathrm{FeCl}_{3}$ at $100{ }^{\circ} \mathrm{C}$ for $10 \mathrm{~min}$, while only $64 \%$ of IBA $2 \mathrm{a}$ was recovered by replacing $\mathrm{H}_{2} \mathrm{SO}_{4}$ in the presence of Oxone ${ }^{\circledR}$ under the same conditions. Thus, the excess uses of Oxone ${ }^{\circledR}$ and performing the reaction at high temperature would decrease the IBA yield as shown in Figure 5a,b.
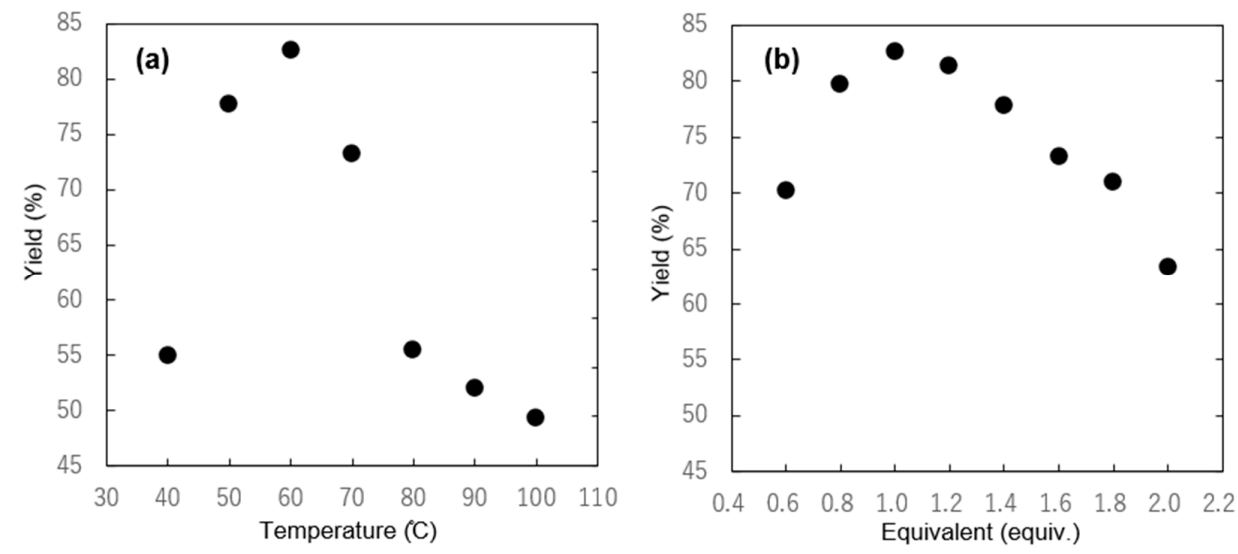

Figure 5. Optimization of (a) temperature using 1.0 equivalent of Oxone ${ }^{\circledR}$ and (b) amount of Oxone ${ }^{\circledR}$ at $60{ }^{\circ} \mathrm{C}$ for the synthesis of IBA $2 \mathrm{a}$ from 2-IB $1 \mathrm{a}$ in the presence of $2.5 \mathrm{~mol} \% \mathrm{FeCl}_{3}(\mathrm{MeCN} / \mathrm{DW}$ $(1 / 1))$. 
This optimized heating method could be applied to the synthesis of IBAs $\mathbf{2 a}-\mathbf{j}$ (Scheme 3). 2-IB $\mathbf{1 a}$ as well as the substrates $\mathbf{1 b}-\mathbf{d}$ and $\mathbf{1 g}-\mathbf{j}$ that are tolerable to over-oxidation at this temperature were successfully converted to the desired IBAs $\mathbf{2} \mathbf{a}-\mathbf{d}$ and $\mathbf{2} \mathbf{g}-\mathbf{j}$ in high yields. However, the transformation of 2-IB having an electron-rich functional group, i.e., methoxysubstituted 2-IB 1f, resulted in low yield of the corresponding IBA $2 \mathbf{f}$ due to the formation of 2-carboxy-p-benzoquinone by the oxidation with Oxone ${ }^{\circledR}$. Therefore, in order to apply the heating conditions, the stability of the product to oxidation must be considered.

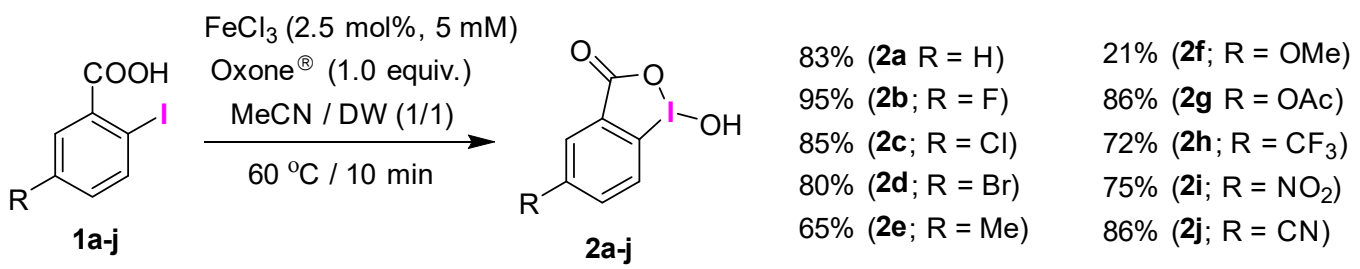

Scheme 3. Short-time selective synthesis of IBAs $\mathbf{2} \mathbf{a}-\mathbf{j}$ in the presence of ferric salt.

\subsection{IBA Derivatives}

As previously mentioned, when IBA 2a was synthesized in an aqueous solution with alcohols, alkoxy-substituted derivatives $\mathbf{3 a}-\mathbf{c}$ were obtained as byproducts by substitution of the hydroxyl ligand of IBA 2a (see Figure 3), implying the potential of the solvolytic ligand exchange of IBA 2a under mild conditions. For the ligand derivatization of IBAs, the water molecule is an obstacle because the ligand exchanges of the IBA hydroxy group are reversible. Thus, molecular sieves with a pore diameter of $3 \AA$ (MS3 $\AA$ ) was used for the solvolytic functionalization of IBA $\mathbf{2 a}$ in dehydrated protic solvent (Figure 6). The quantitative derivatization to benziodoxole methoxide (IB-OMe) 6a was achieved by heating IBA 2a at $60^{\circ} \mathrm{C}$ in $\mathrm{MeOH}$ (Figure 6, entry 1). Upon treatment at $80^{\circ} \mathrm{C}$, benziodoxole ethoxide (IB-OEt) $\mathbf{6 b}$ and benziodoxole 2,2,2-trifluoroethoxide (IB-OCH $\mathrm{CF}_{3}$ ) $\mathbf{6 c}$ were also produced in high yields by the ligand exchange reaction with $\mathrm{EtOH}$ and TFE, respectively (Figure 6, entries 2 and 3). Benziodoxole $n$-propoxide (IB-O ${ }^{n} \operatorname{Pr}$ ) 6d was obtained in 98\% yield using ${ }^{n} \mathrm{PrOH}$ at $70{ }^{\circ} \mathrm{C}$ (Figure 6, entry 4), and benziodoxole isopropoxide (IB-O $\mathrm{Pr}$ ) 6e was produced in $52 \%$ yield at $60{ }^{\circ} \mathrm{C}$ in the presence of ${ }^{i} \mathrm{PrOH}$ (Figure 6 , entry 5). In the cases of IB-O ${ }^{n} \operatorname{Pr} \mathbf{6} \mathbf{d}$ and IB-O $\mathrm{O}^{\mathrm{i}} \operatorname{Pr} \mathbf{6 e}$, the temperature control was essential to suppress the formation of a 2-IB-IBA condensate as a byproduct; here, the formation of 2-IB 1a can be explained in terms of the alcohol oxidation by IBA. It is known that secondary alcohols are readily oxidized by IBA [83]. No unwanted byproduct was found during the transformation to benziodoxole hexafluoroisopropoxide (IB-OCH$\left.\left(\mathrm{CF}_{3}\right)_{2}\right)$ 6f using hexafluoroisopropanol (HFIP) at $80^{\circ} \mathrm{C}$ (Figure 6, entry 6), which is most likely due to the stability of HFIP against oxidation. Indeed, the condensate between 2-IB and IBA appeared during the reaction for benziodoxole $n$-buthoxide (IB-O $\left.{ }^{n} \mathrm{Bu}\right) 6 \mathrm{~g}$ using an oxidizable primary alcohol, ${ }^{n} \mathrm{BuOH}$, at $80{ }^{\circ} \mathrm{C}$, whereas such byproduct was not observed in the synthesis of benziodoxole tert-buthoxide $\left(\mathrm{IB}-\mathrm{O}^{\mathrm{t}} \mathrm{Bu}\right) \mathbf{6 h}$ using ${ }^{t} \mathrm{BuOH}$ as a solvent inert to oxidation. Nevertheless, $\mathrm{IB}^{-} \mathrm{O}^{\mathrm{n}} \mathrm{Bu} 6 \mathrm{~g}$ could be selectively obtained by heat treatment at $60{ }^{\circ} \mathrm{C}$ without the formation of the condensate.

Using $\mathrm{AcOH}$ as a solvent, the solvolytic method was further applied to the synthesis of benziodoxole acetate (IB-OAc) $\mathbf{7 a}$ and its analogs (R-IB-OAc) $\mathbf{7 b} \mathbf{b}-\mathbf{i}$ from the corresponding IBAs 2a-i (Scheme 4). IB-OAc 7a was easily produced in good yield by ligand exchange of IBA 2a with $\mathrm{AcOH}$ at room temperature. Similarly, these transformations successfully afforded R-IB-OAc 7b-d containing fluoro-, chloro-, and bromo-substituents; 7e-g with electron-donating methyl-, methoxy-, and acethoxy-groups; and $7 \mathbf{h}$ and $7 \mathbf{i}$ bearing an electron-withdrawing trifluoromethyl- and nitro-substituent, respectively. 


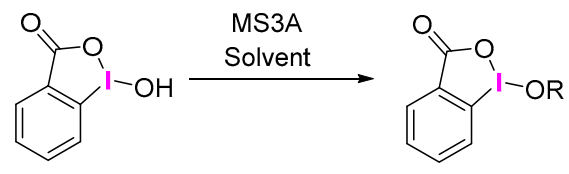

$2 a$

6a-h

\begin{tabular}{cccclc}
\hline Entry & Solvent & Time $(\mathrm{h})$ & Temperature $\left({ }^{\circ} \mathrm{C}\right)$ & Product & Yield (\%) \\
\hline 1 & $\mathrm{MeOH}$ & 2 & 60 & $\mathrm{R}=\mathrm{Me}(\mathbf{6 a})$ & 100 \\
2 & $\mathrm{EtOH}$ & 1 & 80 & $\mathrm{R}=\mathrm{Et}(\mathbf{6 b})$ & 98 \\
3 & $\mathrm{TFE}$ & 1 & 80 & $\mathrm{R}=\mathrm{CH}_{2} \mathrm{CF}_{3}(\mathbf{6 c})$ & 94 \\
4 & ${ }^{n} \mathrm{PrOH}$ & 7 & 70 & $\mathrm{R}={ }^{n} \mathrm{Pr}(\mathbf{6 d})$ & 98 \\
5 & ${ }^{i} \mathrm{PrOH}$ & 15 & 60 & $\mathrm{R}={ }^{i} \mathrm{Pr}(6 \mathrm{e})$ & 52 \\
6 & $\mathrm{HFIP}$ & 1 & $\mathrm{R}=\mathrm{CH}\left(\mathrm{CF}_{3}\right)_{2}(\mathbf{6 f})$ & 83 \\
7 & ${ }^{n} \mathrm{BuOH}$ & 2 & 60 & $\mathrm{R}={ }^{n} \mathrm{Bu}(\mathbf{6 g})$ & 75 \\
8 & ${ }^{t} \mathrm{BuOH}$ & 80 & $\mathrm{R}={ }^{t} \mathrm{Bu}(\mathbf{6 h})$ & 49 \\
\hline
\end{tabular}

Figure 6. Benziodoxole alkoxides $\mathbf{6 a}-\mathbf{h}$ by solvolytic functionalization of IBA $\mathbf{2 a}$.

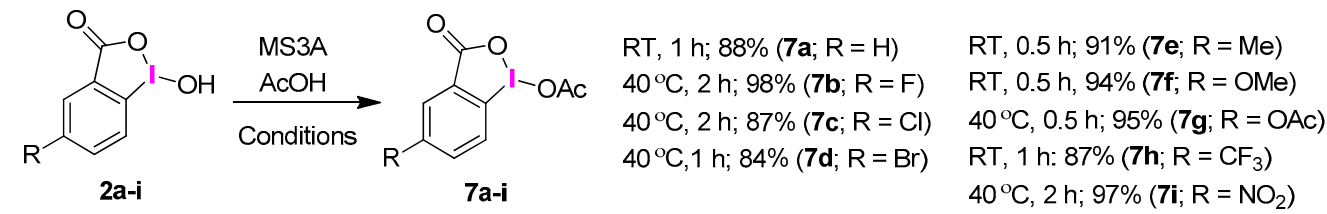

Scheme 4. Transformation of IBAs $2 \mathbf{a}-\mathbf{i}$ to benziodoxole acetates $7 \mathbf{a}-\mathbf{i}$.

\section{Materials and Methods}

\subsection{General Information}

Substrates 1i [96], 1k [97], 1n [98], 1o [98], 1p [99], 1q [100], 1s [101], 1t [98], 1v [102], and 3 [65] were prepared by Sandmeyer reaction of the corresponding anthranilic acids. Substrate $\mathbf{1} \mathbf{g}$ [84] was synthesized by acetylation of compound $\mathbf{1 k}$. Substrate $\mathbf{1} \mathbf{j}$ [103] is derived from 5-bromo-anthranilic acid methyl ester. ${ }^{1} \mathrm{H},{ }^{13} \mathrm{C}$, and ${ }^{19} \mathrm{~F}$ nuclear magnetic resonance (NMR) spectra were recorded on ECS 400 and ECX 500 NMR spectrometers (JEOL Ltd., Tokyo, Japan) using deuterated dimethyl sulfoxide (DMSO-d6) or chloroform $\left(\mathrm{CDCl}_{3}\right)$ as a solvent. Chemical shifts $(\delta)$ are reported in parts per million (ppm) relative to tetramethylsilane $(\delta=0 \mathrm{ppm})$ as an internal standard for ${ }^{1} \mathrm{H}$ and ${ }^{13} \mathrm{C}$ NMR spectra and hexafluoroacetone $(\delta=-84.6 \mathrm{ppm})$ as an internal standard for ${ }^{19} \mathrm{~F}$ NMR spectra. Coupling constants $(J)$ are reported in Hertz $(\mathrm{Hz})$, and the multiplicity is reported according to the following convention: singlet (s), doublet (d), double doublet (dd), double double doublet (ddd), double triplet (dt), triplet (t), triple doublet (td), quartet (q), quintet (quin), sextet (sext), septet (sep), and multiplet (m). Data are reported as follows: Chemical shift (number of protons, multiplicity, coupling constants). Infrared (IR) spectra were recorded on a JASCO FT/IR-4200 spectrometer (JASCO Co., Tokyo, Japan) on diffuse reflectance method using $\mathrm{KBr}$ powder. Absorptions are expressed in reciprocal centimeter $\left(\mathrm{cm}^{-1}\right)$. High resolution mass spectra (HRMS) obtained by the direct analysis in real time (DART) method were recorded on a Thermo Scientific Exactive Plus Orbitrap (Thermo Fisher Scientific, Inc., Waltham, MA, USA).

\subsection{Synthesis of IBA Analogues}

\subsubsection{General Procedure for the Synthesis of IBAs $2 \mathbf{a}-\mathbf{v}$ and 4}

To a solution of 2-IBs $(1.0 \mathrm{mmol})$ in MeCN $(5 \mathrm{~mL})$ was added Oxone ${ }^{\circledR}(738 \mathrm{mg}$, $1.2 \mathrm{mmol}$ ) and $\mathrm{H}_{2} \mathrm{O}$ (TW for Figure 3; Figure 4, Scheme 1 or DW for Scheme 2; Scheme 3, $5 \mathrm{~mL}$ ). After the mixture was stirred at room temperature for the appropriate time (see Figure 4 and Scheme 1), the product was filtered under reduced pressure. The residue was washed with water and acetone to obtain the corresponding IBAs $\mathbf{2 a}-\mathbf{v}$ and $\mathbf{4}$ (see Supplementary Materials for ${ }^{1} \mathrm{H}$ NMR spectroscopic data) as a white powder. 


\subsubsection{1-Hydroxy-1 $1 \lambda^{3}$-benzo[d][1,2]iodaoxol-3(1H)-one (2a)}

${ }^{1} \mathrm{H}$ NMR $(400 \mathrm{MHz}$, DMSO- $d 6): \delta 7.72(1 \mathrm{H}, \mathrm{td}, J=7.3,0.9 \mathrm{~Hz}, \mathrm{H} 5), 7.86(1 \mathrm{H}, \mathrm{d}$, $J=8.2 \mathrm{~Hz}, \mathrm{H} 3), 7.97(1 \mathrm{H}, \mathrm{ddd}, J=8.7,7.4,1.8 \mathrm{~Hz}, \mathrm{H} 4), 8.03(1 \mathrm{H}, \mathrm{dd}, J=7.8,1.4 \mathrm{~Hz}, \mathrm{H6}), 8.08$ (1H, s) ppm. ${ }^{13} \mathrm{C}$ NMR (100 MHz, DMSO-d6): $\delta 120.3$ (C2), 126.2 (C3), 130.3 (C5), 131.0 (C1), 131.4 (C6), $134.4(\mathrm{C} 4), 167.7(\mathrm{COOH}) \mathrm{ppm}$. IR (ATR, KBr): v $2936(\mathrm{OH}), 1616(\mathrm{C}=\mathrm{O}), 1566$ $(\mathrm{C}=\mathrm{O}) \mathrm{cm}^{-1}$. Mp: $243-244{ }^{\circ} \mathrm{C} .{ }^{1} \mathrm{H}$ and ${ }^{13} \mathrm{C}$ NMR data are consistent with those reported in the literature [33].

\subsubsection{5-Fluoro-1-hydroxy-1 $\lambda^{3}$-benzo[d][1,2]iodaoxol-3(1H)-one $(\mathbf{2 b})$}

${ }^{1} \mathrm{H}$ NMR (500 MHz, DMSO- $\left.d 6\right): \delta 7.76(1 \mathrm{H}, \mathrm{dd}, J=8.3,2.6 \mathrm{~Hz}), 7.79-7.88(2 \mathrm{H}, \mathrm{m})$, $8.21(1 \mathrm{H}, \mathrm{s}) \mathrm{ppm} .{ }^{13} \mathrm{C}$ NMR $(125 \mathrm{MHz}$, DMSO-d6): $\delta 114.2,117.3(\mathrm{~d}, J=22.7 \mathrm{~Hz}), 121.7$ $(\mathrm{d}, J=23.8 \mathrm{~Hz}), 128.3(\mathrm{~d}, J=8.4 \mathrm{~Hz}), 134.1(\mathrm{~d}, J=7.2 \mathrm{~Hz}), 163.9(\mathrm{~d}, J=246.8 \mathrm{~Hz}), 166.4(\mathrm{~d}$, $J=2.4 \mathrm{~Hz})$ ppm. ${ }^{19} \mathrm{~F}$ NMR $(470 \mathrm{MHz}, \mathrm{DMSO}-d 6): \delta-116.2(\mathrm{dt}, J=5.7,8.6 \mathrm{~Hz}) \mathrm{ppm}$. IR (ATR, KBr): $v 2904(\mathrm{OH}), 1635(\mathrm{C}=\mathrm{O}), 1577(\mathrm{C}=\mathrm{O}) \mathrm{cm}^{-1}$. Mp: $241-242{ }^{\circ} \mathrm{C} .{ }^{1} \mathrm{H}$ and ${ }^{13} \mathrm{C}$ NMR data are consistent with those reported in the literature [104].

\subsubsection{5-Chloro-1-hydroxy-1 $\lambda^{3}$-benzo[d][1,2]iodaoxol-3(1H)-one (2c)}

${ }^{1} \mathrm{H}$ NMR $(400 \mathrm{MHz}$, DMSO-d6): $\delta 7.81(1 \mathrm{H}, \mathrm{d}, J=8.7 \mathrm{~Hz}), 7.95(1 \mathrm{H}, \mathrm{d}, J=2.3 \mathrm{~Hz}), 8.03$ $(1 \mathrm{H}, \mathrm{dd}, J=8.7,2.3 \mathrm{~Hz}), 8.28(1 \mathrm{H}, \mathrm{s}) \mathrm{ppm} .{ }^{13} \mathrm{C}$ NMR $(100 \mathrm{MHz}, \mathrm{DMSO}-d 6): \delta 118.6,128.1$, 130.3, 133.5, 134.0, 135.8, 166.3 ppm. IR (ATR, KBr): $v 2905(\mathrm{OH}), 1624$ (C=O), $1560(\mathrm{C}=\mathrm{O})$ $\mathrm{cm}^{-1}$. Mp: $294-295{ }^{\circ} \mathrm{C} .{ }^{1} \mathrm{H}$ and ${ }^{13} \mathrm{C}$ NMR data are consistent with those reported in the literature [105].

\subsubsection{5-Bromo-1-hydroxy-1 $\lambda^{3}$-benzo[d][1,2]iodaoxol-3(1H)-one (2d)}

${ }^{1} \mathrm{H}$ NMR $(400 \mathrm{MHz}$, DMSO-d6): $\delta 7.74(1 \mathrm{H}, \mathrm{d}, J=8.7 \mathrm{~Hz}), 8.07(1 \mathrm{H}, \mathrm{d}, J=2.3 \mathrm{~Hz}), 8.15$ $(1 \mathrm{H}, \mathrm{dd}, J=8.7,2.3 \mathrm{~Hz}), 8.27(1 \mathrm{H}, \mathrm{s}) \mathrm{ppm} .{ }^{13} \mathrm{C}$ NMR $(100 \mathrm{MHz}, \mathrm{DMSO}-d 6): \delta 119.5,124.2$, 128.3, 133.3, 133.7, 136.8, 166.2 ppm. IR (ATR, KBr): $v 2884(\mathrm{OH}), 1617(\mathrm{C}=\mathrm{O}), 1557(\mathrm{C}=\mathrm{O})$ $\mathrm{cm}^{-1}$. Mp: $236-238{ }^{\circ} \mathrm{C} .{ }^{1} \mathrm{H}$ and ${ }^{13} \mathrm{C}$ NMR data are consistent with those reported in the literature [106].

\subsubsection{1-Hydroxy-5-methyl-1 $1 \lambda^{3}$-benzo[d][1,2]iodaoxol-3(1H)-one (2e)}

${ }^{1} \mathrm{H}$ NMR (400 MHz, DMSO-d6): $\delta 2.48(3 \mathrm{H}, \mathrm{s}), 7.70(1 \mathrm{H}, \mathrm{d}, J=8.2 \mathrm{~Hz}), 7.79(1 \mathrm{H}, \mathrm{dd}$, $J=8.7,1.8 \mathrm{~Hz}), 7.85(1 \mathrm{H}, \mathrm{s}), 8.01(1 \mathrm{H}, \mathrm{s}) \mathrm{ppm} .{ }^{13} \mathrm{C}$ NMR $(100 \mathrm{MHz}, \mathrm{DMSO}-d 6): \delta 20.1,116.7$, $125.9,131.3,131.4,135.2,140.4,167.7$ ppm. IR (ATR, KBr) : $v 3054(\mathrm{OH}), 1622(\mathrm{C}=\mathrm{O}), 1569$ $(\mathrm{C}=\mathrm{O}) \mathrm{cm}^{-1}$. Mp: $212-214{ }^{\circ} \mathrm{C} .{ }^{1} \mathrm{H}$ and ${ }^{13} \mathrm{C}$ NMR data are consistent with those reported in the literature [105].

\subsubsection{1-Hydroxy-5-methoxy-1 $\lambda^{3}$-benzo[d][1,2]iodaoxol-3(1H)-one (2f)}

${ }^{1} \mathrm{H}$ NMR (400 MHz, DMSO-d6): $\delta 3.89(3 \mathrm{H}, \mathrm{s}), 7.52(1 \mathrm{H}, \mathrm{d}, J=2.7 \mathrm{~Hz}), 7.55(1 \mathrm{H}, \mathrm{dd}$, $J=8.7,2.8 \mathrm{~Hz}), 7.67(1 \mathrm{H}, \mathrm{d}, J=9.2 \mathrm{~Hz}), 8.04(1 \mathrm{H}, \mathrm{s}) \mathrm{ppm} .{ }^{13} \mathrm{C}$ NMR $(100 \mathrm{MHz}, \mathrm{DMSO}-d 6):$ $\delta 55.8,108.9,114.8,121.5,127.0,132.9,161.4,167.4$ ppm. IR (ATR, KBr): $v 2953(\mathrm{OH}), 1620$ $(\mathrm{C}=\mathrm{O}), 1577(\mathrm{C}=\mathrm{O}) \mathrm{cm}^{-1}$. Mp: $217-218{ }^{\circ} \mathrm{C} .{ }^{1} \mathrm{H}$ and ${ }^{13} \mathrm{C}$ NMR data are consistent with those reported in the literature [105].

\subsubsection{1-Hydroxy-3-oxo-1,3-dihydro-1 $\lambda^{3}$-benzo[d][1,2]iodaoxol-5-yl acetate (2g)}

${ }^{1} \mathrm{H}$ NMR $(500 \mathrm{MHz}$, DMSO-d6): $\delta 2.33(3 \mathrm{H}, \mathrm{s}), 7.74(1 \mathrm{H}, \mathrm{dd}, J=8.6,2.3 \mathrm{~Hz}), 7.77(1 \mathrm{H}$, $\mathrm{d}, J=2.3 \mathrm{~Hz}), 7.84(1 \mathrm{H}, \mathrm{d}, J=8.6 \mathrm{~Hz}), 8.16(1 \mathrm{H}, \mathrm{s}) \mathrm{ppm} .{ }^{13} \mathrm{C}$ NMR $(125 \mathrm{MHz}$, DMSO-d6): $\delta$ $20.8,116.1,124.2,127.4,127.9,133.0,152.5,166.8,169.0$ ppm. IR (ATR, KBr): $v 2891(\mathrm{OH})$, $1759(\mathrm{C}=\mathrm{O}), 1604(\mathrm{C}=\mathrm{O}), 1559(\mathrm{C}=\mathrm{O}) \mathrm{cm}^{-1} . \mathrm{Mp}: 207-208^{\circ} \mathrm{C}$. HRMS (DART, $\mathrm{m} / \mathrm{z}$ ) calcd for $\mathrm{C}_{9} \mathrm{H}_{8} \mathrm{IO}_{5}[\mathrm{M}+\mathrm{H}]^{+}: 322.9411$; found: 322.9413 . 
3.2.9. 1-Hydroxy-5-(trifluoromethyl)-1 $1 \lambda^{3}$-benzo[d][1,2]iodaoxol-3(1H)-one (2h)

${ }^{1} \mathrm{H}$ NMR $(500 \mathrm{MHz}$, DMSO- $d 6): \delta 8.08(1 \mathrm{H}, \mathrm{d}, J=8.0 \mathrm{~Hz}), 8.21(1 \mathrm{H}, \mathrm{s}), 8.33(1 \mathrm{H}, \mathrm{d}$, $J=8.1 \mathrm{~Hz}), 8.38(1 \mathrm{H}, \mathrm{s}) \mathrm{ppm} .{ }^{13} \mathrm{C}$ NMR $(125 \mathrm{MHz}, \mathrm{DMSO}-d 6): \delta 123.4(\mathrm{q}, J=271.0 \mathrm{~Hz})$, $125.5,127.1(\mathrm{~d}, J=3.6 \mathrm{~Hz}), 127.9,130.6(\mathrm{~d}, J=2.4 \mathrm{~Hz}), 131.6(\mathrm{q}, J=32.6 \mathrm{~Hz}), 132.9,166.3 \mathrm{ppm}$. ${ }^{19} \mathrm{~F}$ NMR (470 MHz, DMSO-d6): $\delta-64.5$ ppm. IR (ATR, KBr): $v 2854(\mathrm{OH}), 1597(\mathrm{C}=\mathrm{O})$, $1559(\mathrm{C}=\mathrm{O}) \mathrm{cm}^{-1}$. Mp: $233-235{ }^{\circ} \mathrm{C}$. HRMS (DART, $\mathrm{m} / \mathrm{z}$ ) calcd for $\mathrm{C}_{8} \mathrm{H}_{5} \mathrm{~F}_{3} \mathrm{IO}_{3}[\mathrm{M}+\mathrm{H}]^{+}$: 332.9230; found: 332.9227.

\subsubsection{1-Hydroxy-5-nitro- $1 \lambda^{3}$-benzo[d][1,2]iodaoxol-3(1H)-one (2i)}

${ }^{1} \mathrm{H}$ NMR $(400 \mathrm{MHz}$, DMSO- $d 6): \delta 8.10(1 \mathrm{H}, \mathrm{d}, J=8.7 \mathrm{~Hz}), 8.54(1 \mathrm{H}, \mathrm{s}), 8.57(1 \mathrm{H}, \mathrm{d}$, $J=2.3 \mathrm{~Hz}), 8.73(1 \mathrm{H}, \mathrm{dd}, J=8.7,2.8 \mathrm{~Hz}) \mathrm{ppm} .{ }^{13} \mathrm{C}$ NMR $(100 \mathrm{MHz}, \mathrm{DMSO}-d 6): \delta 124.8$, 127.7, 128.1, 128.2, 133.4, 149.7, 165.9 ppm. IR (ATR, KBr) $v 2834(\mathrm{OH}), 1617(\mathrm{C}=\mathrm{O}), 1572$ $(\mathrm{C}=\mathrm{O}), 1541(\mathrm{C}=\mathrm{O}) \mathrm{cm}^{-1}$. Mp: $214-216{ }^{\circ} \mathrm{C} .{ }^{1} \mathrm{H}$ and ${ }^{13} \mathrm{C}$ NMR data are consistent with those reported in the literature [107].

3.2.11. 1-Hydroxy-3-oxo-1,3-dihydro-1 $\lambda^{3}$-benzo[d][1,2]iodaoxole-5-carbonitrile (2j)

${ }^{1} \mathrm{H}$ NMR (500 MHz, DMSO-d6): $\delta 8.01(1 \mathrm{H}, \mathrm{d}, J=8.6 \mathrm{~Hz}), 8.32-8.41(3 \mathrm{H}, \mathrm{m}) \mathrm{ppm} .{ }^{13} \mathrm{C}$ NMR (125 MHz, DMSO-d6): $\delta 113.5,117.3,126.4,127.7,132.9,134.2,137.0,166.0$ ppm. IR (ATR, KBr): $v 2903(\mathrm{OH}), 1625(\mathrm{C}=\mathrm{O}), 1582(\mathrm{C}=\mathrm{O}), 1561(\mathrm{C}=\mathrm{O}) \mathrm{cm}^{-1} . \mathrm{Mp}: 234-236{ }^{\circ} \mathrm{C}$. HRMS (DART, $\mathrm{m} / \mathrm{z}$ ) calcd for $\mathrm{C}_{8} \mathrm{H}_{5} \mathrm{INO}_{3}[\mathrm{M}+\mathrm{H}]^{+}$: 289.9309; found: 289.9310 .

3.2.12. 1,5-Dihydroxy-1 $\lambda^{3}$-benzo[d][1,2]iodaoxol-3(1H)-one (2k)

${ }^{1} \mathrm{H}$ NMR $(400 \mathrm{MHz}$, DMSO-d6): $\delta 7.36(1 \mathrm{H}, \mathrm{dd}, J=8.7,2.7 \mathrm{~Hz}), 7.40(1 \mathrm{H}, \mathrm{d}, J=2.3 \mathrm{~Hz})$, $7.57(1 \mathrm{H}, \mathrm{d}, J=9.2 \mathrm{~Hz}), 7.94(1 \mathrm{H}, \mathrm{s}) \mathrm{ppm} .{ }^{13} \mathrm{C}$ NMR $(100 \mathrm{MHz}, \mathrm{DMSO}-d 6): \delta 106.6,117.1$, 122.0, 127.0, 132.8, 159.7, 167.6 ppm. IR (ATR, KBr): $v 3447(\mathrm{OH}), 3234(\mathrm{OH}), 1576(\mathrm{C}=\mathrm{O})$ $\mathrm{cm}^{-1}$. Mp: $230-232{ }^{\circ} \mathrm{C}$. HRMS (DART, $\mathrm{m} / \mathrm{z}$ ) calcd for $\mathrm{C}_{7} \mathrm{H}_{6} \mathrm{IO}_{4}[\mathrm{M}+\mathrm{H}]^{+}: 280.9305$; found: 280.9304 .

3.2.13. 6-Fluoro-1-hydroxy-1 $\lambda^{3}$-benzo[d][1,2]iodaoxol-3(1H)-one (21)

${ }^{1} \mathrm{H}$ NMR (500 MHz, DMSO- $\left.d 6\right): \delta 7.53-7.60(2 \mathrm{H}, \mathrm{m}), 8.01(1 \mathrm{H}, \mathrm{dd}, J=8.0,5.2 \mathrm{~Hz}), 8.21$ $(1 \mathrm{H}, \mathrm{s}) \mathrm{ppm} .{ }^{13} \mathrm{C}$ NMR $(125 \mathrm{MHz}, \mathrm{DMSO}-d 6): \delta 113.5(\mathrm{~d}, J=27.4 \mathrm{~Hz}), 118.0(\mathrm{~d}, J=22.7 \mathrm{~Hz})$, $122.8(\mathrm{~d}, J=8.4 \mathrm{~Hz}), 128.3,132.9(\mathrm{~d}, J=8.4 \mathrm{~Hz}), 166.0(\mathrm{~d}, J=254.0 \mathrm{~Hz}), 166.7 \mathrm{ppm} .{ }^{19} \mathrm{~F}$ NMR (470 MHz, DMSO-d6): $\delta-109.0(\mathrm{dt}, J=5.8,8.6 \mathrm{~Hz}) \mathrm{ppm}$. IR (ATR, KBr): $v 3091(\mathrm{OH}), 1636$ $(\mathrm{C}=\mathrm{O}), 1586(\mathrm{C}=\mathrm{O}) \mathrm{cm}^{-1}$. Mp: $206-208{ }^{\circ} \mathrm{C} .{ }^{1} \mathrm{H}$ and ${ }^{13} \mathrm{C}$ NMR data are consistent with those reported in the literature [105].

3.2.14. 6-Chloro-1-hydroxy-1 $\lambda^{3}$-benzo[d][1,2]iodaoxol-3(1H)-one (2m)

${ }^{1} \mathrm{H}$ NMR $(400 \mathrm{MHz}$, DMSO- $d 6): \delta 7.75(1 \mathrm{H}, \mathrm{d}, J=1.8 \mathrm{~Hz}), 7.78(1 \mathrm{H}, \mathrm{dd}, J=7.8,1.8 \mathrm{~Hz})$, $7.96(1 \mathrm{H}, \mathrm{d}, J=8.2,2.3 \mathrm{~Hz}), 8.27(1 \mathrm{H}, \mathrm{s}) \mathrm{ppm} .{ }^{13} \mathrm{C}$ NMR $(100 \mathrm{MHz}, \mathrm{DMSO}-\mathrm{d} 6): \delta 122.1,125.7$, 130.6, 130.7, 132.2, 139.3, 166.7 ppm. IR (ATR, KBr): $v 2854(\mathrm{OH}), 1607(\mathrm{C}=\mathrm{O}), 1557(\mathrm{C}=\mathrm{O})$ $\mathrm{cm}^{-1}$. Mp: $212-214{ }^{\circ} \mathrm{C} .{ }^{1} \mathrm{H}$ NMR data is consistent with those reported in the literature [94].

\subsubsection{6-Bromo-1-hydroxy-1 $\lambda^{3}$-benzo[d][1,2]iodaoxol-3(1H)-one (2n)}

${ }^{1} \mathrm{H}$ NMR (500 MHz, DMSO-d6): $\delta 7.80-7.98(3 \mathrm{H}, \mathrm{m}), 8.23(1 \mathrm{H}, \mathrm{s}) \mathrm{ppm} .{ }^{13} \mathrm{C} \mathrm{NMR}$ (125 MHz, DMSO-d6): $\delta 122.1,127.9,128.5,131.0,132.5,133.5,166.8$ ppm. IR (ATR, KBr): $v$ $2844(\mathrm{OH}), 1602(\mathrm{C}=\mathrm{O}), 1556(\mathrm{C}=\mathrm{O}) \mathrm{cm}^{-1}$. Mp: 222-224 ${ }^{\circ} \mathrm{C}$. HRMS (DART, $\left.\mathrm{m} / \mathrm{z}\right)$ calcd for $\mathrm{C}_{7} \mathrm{H}_{5} \mathrm{BrIO}_{3}[\mathrm{M}+\mathrm{H}]^{+}$: 342.8461; found: 342.8460 .

3.2.16. 1-Hydroxy-6-(trifluoromethyl)-1 $\lambda^{3}$-benzo[d][1,2]iodaoxol-3(1H)-one (2o)

${ }^{1} \mathrm{H}$ NMR $(400 \mathrm{MHz}$, DMSO-d6): $\delta 8.06(1 \mathrm{H}, \mathrm{s}), 8.10(1 \mathrm{H}, \mathrm{d}, J=8.1 \mathrm{~Hz}), 8.20(1 \mathrm{H}, \mathrm{d}$, $J=8.0 \mathrm{~Hz}), 8.38(1 \mathrm{H}, \mathrm{s}) \mathrm{ppm} .{ }^{13} \mathrm{C}$ NMR $(100 \mathrm{MHz}, \mathrm{DMSO}-\mathrm{d} 6): \delta 121.7,123.1(\mathrm{~d}, J=3.6 \mathrm{~Hz})$, $123.4(\mathrm{~d}, J=270.7 \mathrm{~Hz}), 127.6(\mathrm{~d}, J=3.6 \mathrm{~Hz}), 131.9,133.9(\mathrm{q}, J=32.2 \mathrm{~Hz}), 135.4,166.4 \mathrm{ppm} .{ }^{19} \mathrm{~F}$ NMR (370 MHz, DMSO-d6): $\delta-64.6$ ppm. IR (ATR, KBr): $v 2871(\mathrm{OH}), 1616(\mathrm{C}=\mathrm{O}), 1560$ 
$(\mathrm{C}=\mathrm{O}) \mathrm{cm}^{-1}$. Mp: $216-217{ }^{\circ} \mathrm{C} .{ }^{1} \mathrm{H}$ and ${ }^{13} \mathrm{C}$ NMR data are consistent with those reported in the literature [12].

3.2.17. 1-Hydroxy-3-oxo-1,3-dihydro-1 $\lambda^{3}$-benzo[d][1,2]iodaoxole-6-carboxylic acid (2p)

${ }^{1} \mathrm{H}$ NMR (500 MHz, DMSO-d6): $\delta$ ppm. ${ }^{13} \mathrm{C}$ NMR (125 MHz, DMSO-d6): $\delta$ ppm. IR (ATR, KBr): $v 2832(\mathrm{OH}), 1704(\mathrm{C}=\mathrm{O}), 1616(\mathrm{C}=\mathrm{O}), 1558(\mathrm{C}=\mathrm{O}) \mathrm{cm}^{-1}$. Mp: 291-293 ${ }^{\circ} \mathrm{C}$. HRMS (DART, $\mathrm{m} / \mathrm{z}$ ) calcd for $\mathrm{C}_{8} \mathrm{H}_{6} \mathrm{IO}_{5}[\mathrm{M}+\mathrm{H}]^{+}$: 308.9254; found: 308.9252.

3.2.18. 5,6-Difluoro-1-hydroxy-1 $1 \lambda^{3}$-benzo[d][1,2]iodaoxol-3(1H)-one (2q)

${ }^{1} \mathrm{H}$ NMR (500 MHz, DMSO-d6): $\delta 7.73(1 \mathrm{H}, \mathrm{dd}, J=9.2,6.9 \mathrm{~Hz}), 7.97(1 \mathrm{H}, \mathrm{dd}, J=9.7$, $7.4 \mathrm{~Hz}), 8.37(1 \mathrm{H}, \mathrm{s})$ ppm. ${ }^{13} \mathrm{C}$ NMR $(125 \mathrm{MHz}, \mathrm{DMSO}-d 6): \delta 115.5(\mathrm{~d}, J=22.7 \mathrm{~Hz}), 119.2(\mathrm{~d}$, $J=19.1 \mathrm{~Hz}), 129.16(\mathrm{~d}, J=2.4 \mathrm{~Hz}), 129.20(\mathrm{~d}, J=3.6 \mathrm{~Hz}), 151.3(\mathrm{dd}, J=263.5,13.1 \mathrm{~Hz}), 153.7$ $(\mathrm{dd}, J=256.4,14.3 \mathrm{~Hz}), 165.9 \mathrm{ppm} .{ }^{19} \mathrm{~F}$ NMR (470 MHz, DMSO- $\left.d 6\right): \delta-138.7-138.5(\mathrm{~m})$, $-132.6-132.4(\mathrm{~m}) \mathrm{ppm}$. IR (ATR, KBr): $v 2895(\mathrm{OH}), 1624(\mathrm{C}=\mathrm{O}), 1591(\mathrm{C}=\mathrm{O}) \mathrm{cm}^{-1}$. Mp: 201-203 ${ }^{\circ} \mathrm{C}$. HRMS (DART, $\mathrm{m} / \mathrm{z}$ ) calcd for $\mathrm{C}_{7} \mathrm{H}_{4} \mathrm{~F}_{2} \mathrm{IO}_{3}[\mathrm{M}+\mathrm{H}]^{+}:$300.9168; found: 300.9170.

3.2.19. 1-Hydroxy-5,6-dimethoxy-1 $\lambda^{3}$-benzo[d][1,2]iodaoxol-3(1H)-one (2r)

${ }^{1} \mathrm{H}$ NMR (500 MHz, DMSO-d6): $\delta 3.89(6 \mathrm{H}, \mathrm{s}), 7.24(1 \mathrm{H}, \mathrm{s}), 7.46(1 \mathrm{H}, \mathrm{s}), 7.95 \mathrm{ppm} .{ }^{13} \mathrm{C}$ NMR (125 MHz, DMSO-d6): $\delta 55.9,56.0,107.4,110.7,112.4,123.9,150.6,154.1,167.8$ ppm. IR (ATR, KBr): $v 3016(\mathrm{OH}), 1592(\mathrm{C}=\mathrm{O}), 1559(\mathrm{C}=\mathrm{O}) \mathrm{cm}^{-1}$. Mp: $201-203{ }^{\circ} \mathrm{C} .{ }^{1} \mathrm{H}$ and ${ }^{13} \mathrm{C}$ NMR data are consistent with those reported in the literature [46].

\subsubsection{7-Bromo-1-hydroxy-1 $\lambda^{3}$-benzo[d][1,2]iodaoxol-3(1H)-one (2s)}

${ }^{1} \mathrm{H}$ NMR $(500 \mathrm{MHz}$, DMSO-d6): $\delta 7.60(1 \mathrm{H}, \mathrm{t}, J=7.7 \mathrm{~Hz}), 7.97(1 \mathrm{H}, \mathrm{dd}, J=7.7$, $1.5 \mathrm{~Hz}), 8.02(1 \mathrm{H}, \mathrm{dd}, J=7.5,1.2 \mathrm{~Hz}) \mathrm{ppm} .{ }^{13} \mathrm{C}$ NMR (125 MHz, DMSO-d6): $\delta 119.6,130.0$, 133.1, 135.4, 140.5, 146.1, 167.0 ppm. IR (ATR, KBr): $v 3273(\mathrm{OH}), 1647(\mathrm{C}=\mathrm{O}) \mathrm{cm}^{-1}$. Mp: 154-155 ${ }^{\circ} \mathrm{C}$. HRMS (DART, m/z) calcd for $\mathrm{C}_{7} \mathrm{H}_{5} \mathrm{BrIO}_{3}[\mathrm{M}+\mathrm{H}]^{+}: 342.8461$; found: 342.8462.

\subsubsection{1-Hydroxy-7-methyl-1 $\lambda^{3}$-benzo[d][1,2]iodaoxol-3(1H)-one (2t)}

${ }^{1} \mathrm{H}$ NMR (400 MHz, DMSO-d6): $\delta 2.79(3 \mathrm{H}, \mathrm{s}), 7.57-7.73(2 \mathrm{H}, \mathrm{m}), 7.90(1 \mathrm{H}, \mathrm{d}, J=6.9 \mathrm{~Hz})$ ppm. ${ }^{13} \mathrm{C}$ NMR (100 MHz, DMSO-d6): $\delta$ 19.6, 128.7, 132.0, 132.6, 137.9, 139.1, 147.4, $167.9 \mathrm{ppm}$. IR (ATR, KBr): $v 1672(\mathrm{C}=\mathrm{O}) \mathrm{cm}^{-1}$. Mp: $164-166{ }^{\circ} \mathrm{C} .{ }^{1} \mathrm{H}$ NMR data are consistent with those reported in the literature [106].

3.2.22. 4-Fluoro-1-hydroxy- $1 \lambda^{3}$-benzo[d][1,2]iodaoxol-3(1H)-one (2u)

${ }^{1} \mathrm{H}$ NMR (400 MHz, DMSO-d6): $\delta 7.51(1 \mathrm{H}, \mathrm{dd}, J=10.1,8.2 \mathrm{~Hz}), 7.71(1 \mathrm{H}, \mathrm{d}, J=7.7 \mathrm{~Hz})$, $7.90(1 \mathrm{H}, \mathrm{td}, J=8.2,4.6 \mathrm{~Hz}), 8.24(1 \mathrm{H}, \mathrm{s}) \mathrm{ppm} .{ }^{13} \mathrm{C}$ NMR $(100 \mathrm{MHz}, \mathrm{DMSO}-d 6): \delta 118.6$ $(\mathrm{d}, J=22.0 \mathrm{~Hz}), 119.2(\mathrm{~d}, J=11.5 \mathrm{~Hz}), 122.5(\mathrm{~d}, J=3.8 \mathrm{~Hz}), 123.2,134.3(\mathrm{~d}, J=8.6 \mathrm{~Hz})$, $163.8(\mathrm{~d}, J=4.8 \mathrm{~Hz}), 163.8(\mathrm{~d}, J=264.4 \mathrm{~Hz}) \mathrm{ppm} .{ }^{19} \mathrm{~F}$ NMR $(375 \mathrm{MHz}, \mathrm{DMSO}-d 6): \delta$ $-114.7(\mathrm{dd}, J=15.2,4.9 \mathrm{~Hz}) \mathrm{ppm}$. IR (ATR, KBr): v $3091(\mathrm{OH}), 1636(\mathrm{C}=\mathrm{O}), 1586(\mathrm{C}=\mathrm{O})$ $\mathrm{cm}^{-1}$. Mp: $213-214{ }^{\circ} \mathrm{C} .{ }^{1} \mathrm{H}$ and ${ }^{13} \mathrm{C}$ NMR data are consistent with those reported in the literature $[106,108]$.

\subsubsection{1-Hydroxy-4-methyl-1 $1 \lambda^{3}$-benzo[d][1,2]iodaoxol-3(1H)-one (2v)}

${ }^{1} \mathrm{H}$ NMR (400 MHz, DMSO-d6): $\delta 2.70(3 \mathrm{H}, \mathrm{s}), 7.48-7.55(1 \mathrm{H}, \mathrm{m}), 7.72-7.80(2 \mathrm{H}, \mathrm{m}), 7.94$ $(1 \mathrm{H}, \mathrm{s}) \mathrm{ppm} .{ }^{13} \mathrm{C}$ NMR $(100 \mathrm{MHz}$, DMSO-d6): $\delta 20.4,122.2,124.2,128.2,133.3,133.4,144.3$, $168.0 \mathrm{ppm}$. IR (ATR, KBr): $v 2926(\mathrm{OH}), 1625(\mathrm{C}=\mathrm{O}), 1584(\mathrm{C}=\mathrm{O}) \mathrm{cm}^{-1} . \mathrm{Mp}: 212-213^{\circ} \mathrm{C} .{ }^{1} \mathrm{H}$ and ${ }^{13} \mathrm{C}$ NMR data are consistent with those reported in the literature [106].

\subsubsection{1-Hydroxy-1 $\lambda^{3}$-naphtho[2,3-d][1,2]iodaoxol-3(1H)-one (4)}

${ }^{1} \mathrm{H}$ NMR (400 MHz, DMSO-d6): $\delta 7.76(2 \mathrm{H}, \mathrm{m}), 8.14-8.33(2 \mathrm{H}, \mathrm{m}), 8.29(1 \mathrm{H}, \mathrm{d}$, $J=8.2 \mathrm{~Hz}), 8.39(1 \mathrm{H}, \mathrm{s}), 8.69(1 \mathrm{H}, \mathrm{s}) \mathrm{ppm} .{ }^{13} \mathrm{C}$ NMR $(100 \mathrm{MHz}, \mathrm{DMSO}-d 6): \delta 115.9,126.3$, $127.8,127.9,128.1,128.9,129.3,131.7,132.8,135.8,167.7$ ppm. IR (ATR, KBr): $v 3053(\mathrm{OH})$, 
$1698(\mathrm{C}=\mathrm{O}), 1607(\mathrm{C}=\mathrm{O}), 1559(\mathrm{C}=\mathrm{O}) \mathrm{cm}^{-1} . \mathrm{Mp}: 164-165^{\circ} \mathrm{C} .{ }^{1} \mathrm{H}$ and ${ }^{13} \mathrm{C}$ NMR data are consistent with those reported in the literature [107].

\subsection{Synthesis of Benziodoxole Alkoxides}

\subsubsection{General Procedure for the Synthesis of Benziodoxole Alkoxides (6)}

To a suspension of IBA $2 \mathbf{a}(264 \mathrm{mg}, 1.0 \mathrm{mmol})$ in an appropriate alcohol $(10 \mathrm{~mL})$ was added MS3 $\AA$ ( $1 \mathrm{~g})$. After the mixture was stirred under the appropriate conditions (see Figure 6), MS3 $\AA$ was filtered using $\mathrm{CH}_{2} \mathrm{Cl}_{2}$, and the solvents were then removed by evaporation. The residue was washed with hexane and filtered to remove the corresponding alcohol completely. The residue was dissolved with $\mathrm{CH}_{2} \mathrm{Cl}_{2}$ and the extract was then filtered through filter paper to remove unreacted substrate. Removal of the solvent by evaporation gave the corresponding benziodoxole alkoxides $\mathbf{6 a}-\mathbf{h}$ as a white powder.

\subsubsection{1-Methoxy-1 $\lambda^{3}$-benzo[d][1,2]iodaoxol-3(1H)-one (6a)}

${ }^{1} \mathrm{H}$ NMR $\left(500 \mathrm{MHz}, \mathrm{CDCl}_{3}\right): \delta 4.29(3 \mathrm{H}, \mathrm{s}), 7.70(1 \mathrm{H}, \mathrm{t}, J=7.7 \mathrm{~Hz}), 7.78(1 \mathrm{H}, \mathrm{d}$, $J=8.1 \mathrm{~Hz}), 7.91(1 \mathrm{H}, \mathrm{ddd}, J=8.6,6.9,1.2 \mathrm{~Hz}), 8.28(1 \mathrm{H}, \mathrm{dd}, J=7.5,1.2 \mathrm{~Hz}) \mathrm{ppm} .{ }^{13} \mathrm{C}$ NMR $\left(125 \mathrm{MHz}, \mathrm{CDCl}_{3}\right): \delta 62.3,118.6,126.0,130.6,131.0,132.9,135.1,168.0 \mathrm{ppm}$. IR (ATR, $\mathrm{KBr}$ ): $v 1653(\mathrm{C}=\mathrm{O}) \mathrm{cm}^{-1}$. Mp: $161-163{ }^{\circ} \mathrm{C} .{ }^{1} \mathrm{H}$ and ${ }^{13} \mathrm{C}$ NMR data are consistent with those reported in the literature $[28,109]$.

\subsubsection{1-Ethoxy-1 $\lambda^{3}$-benzo[d][1,2]iodaoxol-3(1H)-one (6b)}

${ }^{1} \mathrm{H}$ NMR $\left(500 \mathrm{MHz}, \mathrm{CDCl}_{3}\right): \delta 1.35(3 \mathrm{H}, \mathrm{t}, J=6.9 \mathrm{~Hz}), 4.30(2 \mathrm{H}, \mathrm{q}, J=6.9 \mathrm{~Hz}), 7.70(1 \mathrm{H}$, $\mathrm{t}, J=7.4 \mathrm{~Hz}), 7.79(1 \mathrm{H}, \mathrm{d}, J=8.9 \mathrm{~Hz}), 7.89(1 \mathrm{H}, \mathrm{td}, J=8.6,1.8 \mathrm{~Hz}), 8.28(1 \mathrm{H}, \mathrm{dd}, J=7.2,2.0$ Hz) ppm. ${ }^{13} \mathrm{C} \mathrm{NMR}\left(125 \mathrm{MHz}, \mathrm{CDCl}_{3}\right): \delta 19.0,69.9,118.8,125.9,130.7,131.0,132.9,135.0$, $168.0 \mathrm{ppm}$. IR (ATR, KBr): $v 1655(\mathrm{C}=\mathrm{O}) \mathrm{cm}^{-1}$. Mp: $123-125{ }^{\circ} \mathrm{C} .{ }^{1} \mathrm{H}$ and ${ }^{13} \mathrm{C}$ NMR data are consistent with those reported in the literature [109].

\subsubsection{1-(2,2,2-trifluoroethoxy)-1 $\lambda^{3}$-benzo[d][1,2]iodaoxol-3(1H)-one (6c)}

${ }^{1} \mathrm{H}$ NMR $\left(500 \mathrm{MHz}, \mathrm{CDCl}_{3}\right): \delta 4.52(2 \mathrm{H}, \mathrm{q}, J=8.6 \mathrm{~Hz}), 7.74(1 \mathrm{H}, \mathrm{t}, J=7.5 \mathrm{~Hz}), 7.86$ $(1 \mathrm{H}, \mathrm{d}, J=8.0 \mathrm{~Hz}), 7.97(1 \mathrm{H}, \mathrm{ddd}, J=8.0,6.9,1.2 \mathrm{~Hz}), 8.27(1 \mathrm{H}, \mathrm{dd}, J=7.5,1.2 \mathrm{~Hz}) \mathrm{ppm}$. ${ }^{13} \mathrm{C}$ NMR $\left(125 \mathrm{MHz}, \mathrm{CDCl}_{3}\right): \delta 69.7(\mathrm{q}, J=34.2 \mathrm{~Hz}), 119.0,123.5(\mathrm{q}, J=278.2 \mathrm{~Hz}), 126.5$, $129.5,131.4,133.2,135.9,167.9$ ppm. ${ }^{19} \mathrm{~F}$ NMR $\left(470 \mathrm{MHz}, \mathrm{CDCl}_{3}\right): \delta-77.2(\mathrm{q}, J=9.1 \mathrm{~Hz})$ ppm. IR (ATR, KBr): v $1646(\mathrm{C}=\mathrm{O}) \mathrm{cm}^{-1}$. Mp: $139-141{ }^{\circ} \mathrm{C}$. HRMS (DART, $\mathrm{m} / \mathrm{z}$ ) calcd for $\mathrm{C}_{9} \mathrm{H}_{7} \mathrm{~F}_{3} \mathrm{IO}_{3}[\mathrm{M}+\mathrm{H}]^{+}$: 346.9386; found: 346.9384 .

\subsubsection{1-Propoxy-1 $1 \lambda^{3}$-benzo[d][1,2]iodaoxol-3(1H)-one (6d)}

${ }^{1} \mathrm{H} \mathrm{NMR}\left(500 \mathrm{MHz}, \mathrm{CDCl}_{3}\right): \delta 1.02(3 \mathrm{H}, \mathrm{t}, J=7.5 \mathrm{~Hz}), 1.72(2 \mathrm{H}, \mathrm{sext}, J=7.1 \mathrm{~Hz}), 4.20$ $(2 \mathrm{H}, \mathrm{t}, J=6.6 \mathrm{~Hz}), 7.70(1 \mathrm{H}, \mathrm{t}, J=7.2 \mathrm{~Hz}), 7.79(1 \mathrm{H}, \mathrm{d}, J=8.6 \mathrm{~Hz}), 7.89(1 \mathrm{H}, \mathrm{t}, J=7.2 \mathrm{~Hz})$, $8.28(1 \mathrm{H}, \mathrm{d}, J=7.5 \mathrm{~Hz}) \mathrm{ppm} .{ }^{13} \mathrm{C}$ NMR $\left(125 \mathrm{MHz}, \mathrm{CDCl}_{3}\right): \delta 10.1,26.5,76.0,118.9,125.9$, 130.7, 130.9, 132.8, 135.0, 167.9 ppm. IR (ATR, KBr): $v 1651$ (C=O) $\mathrm{cm}^{-1}$. Mp: $146-148{ }^{\circ} \mathrm{C}$. HRMS (DART, $\mathrm{m} / \mathrm{z}$ ) calcd for $\mathrm{C}_{10} \mathrm{H}_{12} \mathrm{IO}_{3}[\mathrm{M}+\mathrm{H}]^{+}$: 306.9826; found: 306.9823 .

\subsubsection{1-Isopropoxy-1 $1 \lambda^{3}$-benzo[d][1,2]iodaoxol-3(1H)-one (6e)}

${ }^{1} \mathrm{H}$ NMR $\left(500 \mathrm{MHz}, \mathrm{CDCl}_{3}\right): \delta 1.36(6 \mathrm{H}, \mathrm{d}, J=6.3 \mathrm{~Hz}), 4.33(1 \mathrm{H}, \mathrm{sep}, J=6.1 \mathrm{~Hz}), 7.69$ $(1 \mathrm{H}, \mathrm{t}, J=7.4 \mathrm{~Hz}), 7.82(1 \mathrm{H}, \mathrm{d}, J=7.5 \mathrm{~Hz}), 7.88(1 \mathrm{H}, \mathrm{td}, J=7.8,1.5 \mathrm{~Hz}), 8.28(1 \mathrm{H}, \mathrm{dd}, J=7.5$, $1.7 \mathrm{~Hz}) \mathrm{ppm} .{ }^{13} \mathrm{C}$ NMR $\left(125 \mathrm{MHz}, \mathrm{CDCl}_{3}\right): \delta 25.2,75.6,119.1,126.0,130.8,130.9,132.7$, 134.8, $168.0 \mathrm{ppm}$. IR (ATR, KBr): $v 1653(\mathrm{C}=\mathrm{O}) \mathrm{cm}^{-1}$. Mp: $253-254{ }^{\circ} \mathrm{C} .{ }^{1} \mathrm{H}$ and ${ }^{13} \mathrm{C}$ NMR data are consistent with those reported in the literature [109].

3.3.7. 1-((1,1,1,3,3,3-hexafluoropropan-2-yl)oxy)-1 $\lambda^{3}$-benzo[d][1,2]iodaoxol-3(1H)-one (6f)

${ }^{1} \mathrm{H}$ NMR $\left(500 \mathrm{MHz}, \mathrm{CDCl}_{3}\right): \delta 4.80(1 \mathrm{H}, \mathrm{sep}, J=5.8 \mathrm{~Hz}), 7.74(1 \mathrm{H}, \mathrm{ddd}, J=8.0,7.5,1.4$ $\mathrm{Hz}), 7.97-8.04(2 \mathrm{H}, \mathrm{m}), 8.24(1 \mathrm{H}, \mathrm{dd}, J=7.4,1.2 \mathrm{~Hz}) \mathrm{ppm} .{ }^{13} \mathrm{C} \mathrm{NMR}\left(125 \mathrm{MHz}, \mathrm{CDCl}_{3}\right)$ : $\delta 76.1$ (quin, $J=32.5 \mathrm{~Hz}), 119.4,122.2(\mathrm{q}, J=283.8 \mathrm{~Hz}), 127.3,128.5,131.6,133.3,136.4$, 
168.1 ppm. ${ }^{19} \mathrm{~F}$ NMR $\left(470 \mathrm{MHz}, \mathrm{CDCl}_{3}\right): \delta-76.0(\mathrm{~d}, J=5.7 \mathrm{~Hz}) \mathrm{ppm}$. IR (ATR, KBr): $v$ $1661(\mathrm{C}=\mathrm{O}) \mathrm{cm}^{-1}$. Mp: $148-149^{\circ} \mathrm{C}$. HRMS (DART, m/z) calcd for $\mathrm{C}_{10} \mathrm{H}_{6} \mathrm{~F}_{6} \mathrm{IO}_{3}[\mathrm{M}+\mathrm{H}]^{+}$: 414.9260; found: 414.9258 .

\subsubsection{1-Butoxy-1 $\lambda^{3}$-benzo[d][1,2]iodaoxol-3(1H)-one (6g)}

${ }^{1} \mathrm{H} \mathrm{NMR}\left(500 \mathrm{MHz}, \mathrm{CDCl}_{3}\right): \delta 0.98(3 \mathrm{H}, \mathrm{t}, J=7.5 \mathrm{~Hz}), 1.46(2 \mathrm{H}, \mathrm{sext}, J=7.4 \mathrm{~Hz}), 1.68$ $(2 \mathrm{H}$, quin, $J=7.2 \mathrm{~Hz}), 4.24(2 \mathrm{H}, \mathrm{t}, J=6.6 \mathrm{~Hz}), 7.70(1 \mathrm{H}, \mathrm{t}, J=7.5 \mathrm{~Hz}), 7.78(1 \mathrm{H}, \mathrm{d}, J=8.0$ $\mathrm{Hz}), 7.89(1 \mathrm{H}, \mathrm{t}, J=7.5 \mathrm{~Hz}), 8.28(1 \mathrm{H}, \mathrm{d}, J=7.5 \mathrm{~Hz}) \mathrm{ppm} .{ }^{13} \mathrm{C} \mathrm{NMR}\left(125 \mathrm{MHz}, \mathrm{CDCl}_{3}\right): \delta$ $13.9,19.0,35.4,74.2,118.9,125.9,130.7,131.0,132.9,135.0,167.9$ ppm. IR (ATR, KBr): $v 1650$ $(\mathrm{C}=\mathrm{O}) \mathrm{cm}^{-1}$. Mp: $143-144{ }^{\circ} \mathrm{C} .{ }^{1} \mathrm{H}$ and ${ }^{13} \mathrm{C}$ NMR data are consistent with those reported in the literature [110].

\subsubsection{1-(tert-Butoxy)-1 $\lambda^{3}$-benzo[d][1,2]iodaoxol-3(1H)-one (6h)}

${ }^{1} \mathrm{H}$ NMR $\left(500 \mathrm{MHz}, \mathrm{CDCl}_{3}\right): \delta 1.41(9 \mathrm{H}, \mathrm{s}), 7.67(1 \mathrm{H}, \mathrm{ddd}, J=8.1,7.5,1.8 \mathrm{~Hz}), 7.83-7.91$ $(2 \mathrm{H}, \mathrm{m}), 8.26(1 \mathrm{H}, \mathrm{dd}, J=8.1,1.8 \mathrm{~Hz}) \mathrm{ppm} .{ }^{13} \mathrm{C}$ NMR $\left(125 \mathrm{MHz}, \mathrm{CDCl}_{3}\right): \delta 30.4,78.6,119.6$, 126.2, 130.8, 131.0, 132.4, 134.7, 168.0 ppm. IR (ATR, KBr): $v 1659(\mathrm{C}=\mathrm{O}) \mathrm{cm}^{-1}$. Decomp: $265{ }^{\circ} \mathrm{C}$. HRMS (DART, $\mathrm{m} / \mathrm{z}$ ) calcd for $\mathrm{C}_{11} \mathrm{H}_{13} \mathrm{IO}_{3}[\mathrm{M}+\mathrm{H}]^{+}:$320.9982; found: 320.9980.

\subsection{Synthesis of Benziodoxole Acetates}

3.4.1. General Procedure for the Synthesis of Benziodoxole Acetates (7)

MS3A ( $0.5 \mathrm{~g})$ was added to a suspension of IBAs $\mathbf{2 a}-\mathbf{i}(0.50 \mathrm{mmol})$ in $\mathrm{AcOH}(5 \mathrm{~mL})$, and the mixture was stirred under the appropriate conditions (see Scheme 4). Then, MS3A was filtered using $\mathrm{CH}_{2} \mathrm{Cl}_{2}$, and the solvents were removed by evaporation. The residue was washed with ether and filtered to remove $\mathrm{AcOH}$ completely. The resulting residue was dissolved with $\mathrm{CH}_{2} \mathrm{Cl}_{2}$, and the extract was then filtered through filter paper to remove unreacted substrate. After solvent removal by evaporation, the corresponding benziodoxole acetates $7 \mathbf{a}-\mathbf{i}$ were obtained as a white powder.

\subsubsection{3-Oxo-1 $\lambda^{3}$-benzo[d][1,2]iodaoxol-1(3H)-yl acetate (7a)}

${ }^{1} \mathrm{H}$ NMR $\left(500 \mathrm{MHz}, \mathrm{CDCl}_{3}\right): \delta 2.27(3 \mathrm{H}, \mathrm{s}), 7.72(1 \mathrm{H}, \mathrm{td}, J=7.5,1.2 \mathrm{~Hz}), 7.94(1 \mathrm{H}, \mathrm{ddd}$, $J=8.6,6.9,1.2 \mathrm{~Hz}), 8.01(1 \mathrm{H}, \mathrm{d}, J=8.6 \mathrm{~Hz}), 8.25(1 \mathrm{H}, \mathrm{dd}, J=7.5,1.2 \mathrm{~Hz}) \mathrm{ppm} .{ }^{13} \mathrm{C}$ NMR (125 MHz, $\mathrm{CDCl}_{3}$ ): $\delta 20.2,118.3,128.9,129.2,131.2,133.1,136.1,168.1,176.3$ ppm. IR (ATR, $\mathrm{KBr}): v 1684(\mathrm{C}=\mathrm{O}) \mathrm{cm}^{-1}$. Mp: $220-222{ }^{\circ} \mathrm{C} .{ }^{1} \mathrm{H}$ and ${ }^{13} \mathrm{C}$ NMR data are consistent with those reported in the literature [109].

\subsubsection{5-Fluoro-3-oxo- $1 \lambda^{3}$-benzo[d][1,2]iodaoxol-1(3H)-yl acetate $(\mathbf{7 b})$}

${ }^{1} \mathrm{H}$ NMR $\left(500 \mathrm{MHz}, \mathrm{CDCl}_{3}\right): \delta 2.26(3 \mathrm{H}, \mathrm{s}), 7.64(1 \mathrm{H}, \mathrm{ddd}, J=8.6,7.7,2.9 \mathrm{~Hz}), 7.94-8.00$ $(2 \mathrm{H}, \mathrm{m}) \mathrm{ppm} .{ }^{13} \mathrm{C} \mathrm{NMR}\left(125 \mathrm{MHz}, \mathrm{CDCl}_{3}\right): \delta 20.2,111.3,120.0(\mathrm{~d}, J=23.8 \mathrm{~Hz}), 123.7(\mathrm{~d}$, $J=22.7 \mathrm{~Hz}), 131.0(\mathrm{~d}, J=8.3 \mathrm{~Hz}), 131.8(\mathrm{~d}, J=7.2 \mathrm{~Hz}), 165.0(\mathrm{~d}, J=252.8 \mathrm{~Hz}), 166.8,176.4$ ppm. ${ }^{19} \mathrm{~F} \mathrm{NMR}\left(470 \mathrm{MHz}, \mathrm{CDCl}_{3}\right): \delta-110.8(\mathrm{td}, J=7.2,4.3 \mathrm{~Hz}) \mathrm{ppm}$. IR (ATR, KBr): $v 1696$ $(\mathrm{C}=\mathrm{O}) \mathrm{cm}^{-1}$. Mp: $225-226{ }^{\circ} \mathrm{C} .{ }^{1} \mathrm{H}$ and ${ }^{13} \mathrm{C}$ NMR data are consistent with those reported in the literature [111].

\subsubsection{5-Chloro-3-oxo-1 $\lambda^{3}$-benzo[d][1,2]iodaoxol-1(3H)-yl acetate (7c)}

${ }^{1} \mathrm{H}$ NMR $\left(500 \mathrm{MHz}, \mathrm{CDCl}_{3}\right): \delta 2.27(3 \mathrm{H}, \mathrm{s}), 7.87(1 \mathrm{H}, \mathrm{dd}, J=8.6,2.3 \mathrm{~Hz}), 7.93(1 \mathrm{H}$, $\mathrm{d}, J=9.2 \mathrm{~Hz}), 8.22(1 \mathrm{H}, \mathrm{d}, J=1.7 \mathrm{~Hz}) \mathrm{ppm} .{ }^{13} \mathrm{C} \mathrm{NMR}\left(125 \mathrm{MHz}, \mathrm{CDCl}_{3}\right): \delta 20.2,115.5$, 130.4, 130.9, 133.0, 136.0, 138.8, 166.7, 176.4 ppm. IR (ATR, KBr): $v 1698(\mathrm{C}=\mathrm{O}) \mathrm{cm}^{-1}$. Mp: 244-245 ${ }^{\circ} \mathrm{C} .{ }^{1} \mathrm{H}$ and ${ }^{13} \mathrm{C}$ NMR data are consistent with those reported in the literature [105].

3.4.5. 5-Bromo-3-oxo- $1 \lambda^{3}$-benzo[d][1,2]iodaoxol-1(3H)-yl acetate (7d)

${ }^{1} \mathrm{H}$ NMR $\left(500 \mathrm{MHz}, \mathrm{CDCl}_{3}\right): \delta 2.27(3 \mathrm{H}, \mathrm{s}), 7.85(1 \mathrm{H}, \mathrm{d}, J=9.2 \mathrm{~Hz}), 8.01(1 \mathrm{H}, \mathrm{dd}, J=8.6$, $2.3 \mathrm{~Hz}), 8.37(1 \mathrm{H}, \mathrm{d}, J=1.8 \mathrm{~Hz}) \mathrm{ppm} .{ }^{13} \mathrm{C} \mathrm{NMR}\left(125 \mathrm{MHz}, \mathrm{CDCl}_{3}\right): \delta 20.2,116.5,126.6,130.7$, 
131.0, 136.1, 138.9, 166.6, 176.4 ppm. IR (ATR, KBr): $v 1680$ (C=O) $\mathrm{cm}^{-1}$. Mp: 226-228 ${ }^{\circ} \mathrm{C}$. ${ }^{1} \mathrm{H}$ and ${ }^{13} \mathrm{C}$ NMR data are consistent with those reported in the literature [112].

3.4.6. 5-Methyl-3-oxo-1 $1 \lambda^{3}$-benzo[d][1,2]iodaoxol-1(3H)-yl acetate (7e)

${ }^{1} \mathrm{H} \mathrm{NMR}\left(500 \mathrm{MHz}, \mathrm{CDCl}_{3}\right): \delta 2.25(3 \mathrm{H}, \mathrm{s}), 2.56(3 \mathrm{H}, \mathrm{s}), 7.73(1 \mathrm{H}, \mathrm{dd}, J=8.6,1.7 \mathrm{~Hz})$, $7.84(1 \mathrm{H}, \mathrm{d}, J=8.6 \mathrm{~Hz}), 8.07(1 \mathrm{H}, \mathrm{d}, J=1.8 \mathrm{~Hz}) \mathrm{ppm} .{ }^{13} \mathrm{C} \mathrm{NMR}\left(125 \mathrm{MHz}, \mathrm{CDCl}_{3}\right): \delta 20.3$, 20.8, 114.6, 128.9, 133.6, 137.1, 142.3, 168.3, 176.4 ppm. IR (ATR, KBr): $v 1682$ (C=O), 1659 $(\mathrm{C}=\mathrm{O}) \mathrm{cm}^{-1}$. Mp: $215-217{ }^{\circ} \mathrm{C} .{ }^{1} \mathrm{H}$ and ${ }^{13} \mathrm{C}$ NMR data are consistent with those reported in the literature [105].

3.4.7. 5-Methoxy-3-oxo-1 $\lambda^{3}$-benzo[d][1,2]iodaoxol-1(3H)-yl acetate $\mathbf{( 7 f )}$

${ }^{1} \mathrm{H}$ NMR $\left(500 \mathrm{MHz}, \mathrm{CDCl}_{3}\right): \delta 2.25(3 \mathrm{H}, \mathrm{s}), 3.94(3 \mathrm{H}, \mathrm{s}), 7.46(1 \mathrm{H}, \mathrm{dd}, J=9.2,2.9 \mathrm{~Hz})$, $7.74(1 \mathrm{H}, \mathrm{d}, J=2.9 \mathrm{~Hz}), 7.81(1 \mathrm{H}, \mathrm{d}, J=8.6 \mathrm{~Hz}) \mathrm{ppm} .{ }^{13} \mathrm{C} \mathrm{NMR}\left(125 \mathrm{MHz}, \mathrm{CDCl}_{3}\right): \delta 20.3$, $56.2,106.8,115.9,124.5,129.7,130.6,162.7,168.1,176.4$ ppm. IR (ATR, KBr): $v 1697$ (C=O), $1681(\mathrm{C}=\mathrm{O}), 1656(\mathrm{C}=\mathrm{O}) \mathrm{cm}^{-1}$. Mp: $207-209^{\circ} \mathrm{C} .{ }^{1} \mathrm{H}$ and ${ }^{13} \mathrm{C}$ NMR data are consistent with those reported in the literature [105].

3.4.8. 3-Oxo-1 $\lambda^{3}$-benzo[d] [1,2]iodaoxole-1,5(3H)-diyl diacetate $(7 \mathrm{~g})$

${ }^{1} \mathrm{H}$ NMR $\left(500 \mathrm{MHz}, \mathrm{CDCl}_{3}\right): \delta 2.26(3 \mathrm{H}, \mathrm{s}), 2.37(3 \mathrm{H}, \mathrm{s}), 7.68(1 \mathrm{H}, \mathrm{dd}, J=8.9,2.6 \mathrm{~Hz})$, 7.96-8.02 (2H, m) ppm. ${ }^{13} \mathrm{C}$ NMR $\left(125 \mathrm{MHz}, \mathrm{CDCl}_{3}\right): \delta 20.3,21.1,113.6,126.2,129.8,130.3$, $130.9,153.6,167.2,168.7,176.5$ ppm. IR (ATR, KBr): $v 1690(\mathrm{C}=\mathrm{O}) \mathrm{cm}^{-1}$. Mp: $153-154^{\circ} \mathrm{C}$. HRMS (DART, $\mathrm{m} / \mathrm{z}$ ) calcd for $\mathrm{C}_{11} \mathrm{H}_{10} \mathrm{IO}_{6}[\mathrm{M}+\mathrm{H}]^{+}$: 364.9517; found: 364.9518 .

3.4.9. 3-Oxo-5-(trifluoromethyl)-1 $1 \lambda^{3}$-benzo[d][1,2]iodaoxol-1(3H)-yl acetate (7h)

${ }^{1} \mathrm{H}$ NMR $\left(500 \mathrm{MHz}, \mathrm{CDCl}_{3}\right): \delta 2.29(3 \mathrm{H}, \mathrm{s}), 8.14(1 \mathrm{H}, \mathrm{dd}, J=8.6,1.7 \mathrm{~Hz}), 8.20(1 \mathrm{H}, \mathrm{d}$, $J=8.6 \mathrm{~Hz}), 8.52(1 \mathrm{H}, \mathrm{d}, J=1.7 \mathrm{~Hz}) \mathrm{ppm} .{ }^{13} \mathrm{C} \mathrm{NMR}\left(125 \mathrm{MHz}, \mathrm{CDCl}_{3}\right): \delta 20.2,121.9,122.8$ $(\mathrm{q}, J=271.4 \mathrm{~Hz}), 130.1(\mathrm{~d}, J=3.6 \mathrm{~Hz}), 130.4(\mathrm{~d}, J=9.5 \mathrm{~Hz}), 132.4(\mathrm{~d}, J=2.4 \mathrm{~Hz}), 134.5(\mathrm{q}$, $J=33.8 \mathrm{~Hz}), 166.7,176.5 \mathrm{ppm} .{ }^{19} \mathrm{~F} \mathrm{NMR}\left(470 \mathrm{MHz}, \mathrm{CDCl}_{3}\right): \delta-64.9 \mathrm{ppm}$. IR (ATR, KBr): $v$ $1692(\mathrm{C}=\mathrm{O}), 1647(\mathrm{C}=\mathrm{O}) \mathrm{cm}^{-1}$. Mp: 212-213 ${ }^{\circ} \mathrm{C}$. HRMS (DART, m/z) calcd for $\mathrm{C}_{10} \mathrm{H}_{7} \mathrm{~F}_{3} \mathrm{IO}_{4}$ $[\mathrm{M}+\mathrm{H}]^{+}$: 374.9336; found: 374.9334 .

\subsubsection{5-Nitro-3-oxo- $1 \lambda^{3}$-benzo[d][1,2]iodaoxol-1(3H)-yl acetate (7i)}

${ }^{1} \mathrm{H}$ NMR $\left(500 \mathrm{MHz}, \mathrm{CDCl}_{3}\right): \delta 2.30(3 \mathrm{H}, \mathrm{s}), 8.27(1 \mathrm{H}, \mathrm{d}, J=9.2 \mathrm{~Hz}), 8.71(1 \mathrm{H}, \mathrm{dd}, J=9.0$, $2.5 \mathrm{~Hz}), 9.04(1 \mathrm{H}, \mathrm{d}, J=2.3 \mathrm{~Hz}) \mathrm{ppm} .{ }^{13} \mathrm{C} \mathrm{NMR}\left(125 \mathrm{MHz}, \mathrm{CDCl}_{3}\right): \delta 20.2,124.1,127.6,129.8$, 131.0, 131.5,150.8, 165.7, 176.6 ppm. IR (ATR, KBr): $v 1705(\mathrm{C}=\mathrm{O}), 1665(\mathrm{C}=\mathrm{O}) \mathrm{cm}^{-1}$. Mp: 209-210 ${ }^{\circ} \mathrm{C} .{ }^{1} \mathrm{H}$ and ${ }^{13} \mathrm{C}$ NMR data are consistent with those reported in the literature [107].

\section{Conclusions}

We have presented a practical synthetic method for IBA from 2-IB without contamination by hazardous pentavalent IBX using cost-effective Oxone ${ }^{\circledR}$ in aqueous solution. This highly safe, convenient method operates under mild conditions such as room temperature, which contrasts with traditional method using reflux conditions and expensive $\mathrm{NaIO}_{4}$ in $\mathrm{AcOH}$ solution. The use of mild conditions circumvents the problem of the formation of byproducts such as potentially explosive pentavalent cyclic hypervalent iodine compound, i.e., IBX; the contamination of IBX into IBA is generally not desired for safety reasons. The reaction time can be shortened by heating; in this case, addition of a ferric salt in our reaction system can effectively suppress the formation of IBX as byproducts. In addition, a convenient derivatization of the hydroxy group of IBAs by solvolytic treatment is presented. These derivatizations were generally achieved under mild conditions below $80^{\circ} \mathrm{C}$. Our methods, which do not require any chromatography technique, can be performed safely and would be suitable for large-scale synthesis.

Supplementary Materials: Supplementary materials are available online, ${ }^{1} \mathrm{H}$ NMR spectroscopic data for the compounds $\mathbf{2 a}-\mathbf{v}$ and $\mathbf{4}$. 
Author Contributions: H.C. found the selective reaction to obtain IBAs using Oxone ${ }^{\circledR}$ and the solvolytic functionalization for IBA and drafted the manuscript; N.K., H.Y., and N.T. also contributed to the experiments; T.D. directed this study as a project and finalized the manuscript with critical discussion. All authors have read and agreed to the published version of the manuscript.

Funding: This work was financially supported by a Grant-in-Aid for Scientific Research (C) (JSPS KAKENHI Grant Number JP19K05466) from JSPS and the Ritsumeikan Global Innovation Research Organization (R-GIRO) project. H.C. also acknowledges support from a Grant-in-Aid for Early-Career Scientists (JSPS KAKENHI Grant Number 20K15103) from JSPS.

Institutional Review Board Statement: Not applicable.

Informed Consent Statement: Not applicable.

Data Availability Statement: The data presented in this study are available in the communication.

Conflicts of Interest: The authors declare no conflict of interest.

Sample Availability: Samples of the compounds are not available from the authors.

\section{References}

1. Zhdankin, V.V. Organoiodine(V) reagents in organic synthesis. J. Org. Chem. 2011, 76, 1185-1197. [CrossRef]

2. Singh, F.V.; Wirth, T. Hypervalent iodine-catalyzed oxidative functionalizations including stereoselective reactions. Chem. Asian J. 2014, 9, 950-971. [CrossRef] [PubMed]

3. Yoshimura, A.; Zhdankin, V.V. Advances in synthetic applications of hypervalent iodine compounds. Chem. Rev. 2016, 116, 3328-3435. [CrossRef] [PubMed]

4. Tan, H.; Ji, W.; Wang, L. Sunlight-driven decarboxylative alkynylation of $\alpha$-keto acids with bromoacetylenes by hypervalent iodine reagent catalysis: A facile approach to ynones. Angew. Chem. Int. Ed. 2015, 54, 8374-8377. [CrossRef] [PubMed]

5. Wang, D.; Zhang, L.; Luo, S. Enantioselective decarboxylative $\alpha$-alkynylation of $\beta$-ketocarbonyls via a catalytic $\alpha$-imino radical Intermediate. Org. Lett. 2017, 19, 4924-4927. [CrossRef]

6. Yang, S.; Tan, H.; Ji, W.; Zhang, X.; Li, P. Visible light-induced decarboxylative acylarylation of phenyl propiolates with $\alpha-$ oxocarboxylic acids to coumarins catalyzed by hypervalent iodine reagents under transition metal-free conditions. Adv. Synth. Catal. 2017, 359, 443-453. [CrossRef]

7. Sun, X.; Liu, T.; Yang, Y.-T.; Gu, Y.-J.; Liu, Y.W.; Ji, Y.G.; Luo, K.; Zhu, J.; Wu, L. Visible-light-promoted regio- and stereoselective oxyalkenylation of phosphinyl allenes. Adv. Synth. Catal. 2020, 362, 2701-2708. [CrossRef]

8. Ball, L.T.; Lloyd-Jones, G.C.; Russell, C.A. Gold-catalysed oxyarylation of styrenes and mono- and gem-disubstituted olefins facilitated by an iodine(III) oxidant. Chem. Eur. J. 2012, 18, 2931-2937. [CrossRef] [PubMed]

9. Hata, K.; Segawa, Y.; Itami, K. Pyridylidene ligand facilitates gold-catalyzed oxidative C-H arylation of heterocycles. Beilstein. J. Org. Chem. 2015, 11, 2737-2746. [CrossRef] [PubMed]

10. Hu, J.; Lan, T.; Sun, Y.; Chen, H.; Yao, J.; Rao, Y. Unactivated $\mathrm{C}\left(\mathrm{sp}^{3}\right)$-H hydroxylation through palladium catalysis with $\mathrm{H}_{2} \mathrm{O}$ as the oxygen source. Chem. Commun. 2015, 51, 14929-14932. [CrossRef] [PubMed]

11. Bindu, V.H.; Parvathaneni, S.P.; Rao, V.J. Iodosobenzoic acid (IBA) catalysed benzylic and aromatic C-H oxidations. Catal. Lett. 2017, 147, 1434-1440. [CrossRef]

12. Li, G.-X.; Morales-Rivera, C.A.; Gao, F.; Wang, Y.; He, G.; Liu, P.; Chen, G. A unified photoredox-catalysis strategy for C(sp $\left.{ }^{3}\right)-H$ hydroxylation and amidation using hypervalent iodine. Chem. Sci. 2017, 8, 7180-7185. [CrossRef]

13. Wang, D.; Ren, R.; Zhu, C. Manganese-promoted ring-opening hydrazination of cyclobutanols: Synthesis of alkyl hydrazines. J. Org. Chem. 2016, 81, 8043-8049. [CrossRef]

14. Ye, L.; Gu, Q.-S.; Tian, Y.; Meng, X.; Chen, G.-C.; Liu, X.-Y. Radical asymmetric intramolecular $\alpha$-cyclopropanation of aldehydes towards bicyclo[3.1.0]hexanes containing vicinal all-carbon quaternary stereocenters. Nat. Commun. 2018, 9, 1-13. [CrossRef]

15. Amos, S.G.E.; Nicolai, S.; Waser, J. Photocatalytic umpolung of $N$ - and $O$-substituted alkenes for the synthesis of 1,2-amino alcohols and diols. Chem. Sci. 2020, 11, 11274-11279. [CrossRef]

16. He, X.-K.; Lu, J.; Zhang, A.-J.; Zhang, Q.-Q.; Xu, G.-Y.; Xuan, J. Bi-OAc-accelerated C3-H alkylation of quinoxaline-2(1H)-ones under visible-light irradiation. Org. Lett. 2020, 22, 5984-5989. [CrossRef]

17. Li, J.; Liu, Z.; Wu, S.; Chen, Y. Acyl radical smiles rearrangement to construct hydroxybenzophenones by photoredox catalysis. Org. Lett. 2019, 21, 2077-2080. [CrossRef] [PubMed]

18. Fang, Z.; Wang, Y.; Wang, Y. Synthesis of 4-iodoisoquinolin-1(2H)-ones by a dirhodium (II)-catalyzed 1,4-bisfunctionalization of isoquinolinium iodide salts. Org. Lett. 2019, 21, 434-438. [CrossRef] [PubMed]

19. Wu, L.; Hao, Y.; Lin, Y.; Wang, Q. Visible-light-induced intramolecular sp ${ }^{3}-\mathrm{C}-\mathrm{H}$ oxidation of 2-alkyl-substituted benzamides for the synthesis of functionalized iminoisobenzofurans. Chem. Commun. 2019, 55, 13908-13911. [CrossRef] [PubMed]

20. Wang, N.; Gu, Q.-S.; Li, Z.-L.; Li, Z.; Guo, Y.-L.; Guo, Z.; Liu, X.-Y. Direct photocatalytic synthesis of medium-sized lactams by C-C bond cleavage. Angew. Chem. Int. Ed. 2018, 57, 14225-14229. [CrossRef] [PubMed] 
21. Pawar, G.G.; Robert, F.; Grau, E.; Cramail, H.; Landais, Y. Visible-light photocatalyzed oxidative decarboxylation of oxamic acids: A green route to urethanes and ureas. Chem. Commun. 2018, 54, 9337-9340. [CrossRef] [PubMed]

22. Jia, K.; Zhang, F.; Huang, H.; Chen, Y. Visible-light-induced alkoxyl radical generation enables selective $\mathrm{C}\left(\mathrm{sp}^{3}\right)-\mathrm{C}\left(\mathrm{sp}^{3}\right)$ bond cleavage and functionalizations. J. Am. Chem. Soc. 2016, 138, 1514-1517. [CrossRef]

23. Li, G.-X.; Morales-Rivera, C.A.; Wang, Y.; Gao, F.; He, G.; Liu, P.; Chen, G. Photoredox-mediated Minisci C-H alkylation of $N$-heteroarenes using boronic acids and hypervalent iodine. Chem. Sci. 2016, 7, 6407-6412. [CrossRef]

24. Ji, W.; Tan, H.; Wang, M.; Li, P.; Wang, L. Photocatalyst-free hypervalent iodine reagent catalyzed decarboxylative acylarylation of acrylamides with $\alpha$-oxocarboxylic acids driven by visible-light irradiation. Chem. Commun. 2016, 52, 1462-1465. [CrossRef] [PubMed]

25. Dai, J.-J.; Zhang, W.-M.; Shu, Y.-J.; Sun, Y.-Y.; Xu, J.; Feng, Y.-S.; Xu, H.-J. Deboronative cyanation of potassium alkyltrifluoroborates via photoredox catalysis. Chem. Commun. 2016, 52, 6793-6796. [CrossRef] [PubMed]

26. Huang, H.; Zhang, G.; Chen, Y. Dual hypervalent iodine (III) reagents and photoredox catalysis enable decarboxylative ynonylation under mild conditions. Angew. Chem. Int. Ed. 2015, 54, 7872-7876. [CrossRef] [PubMed]

27. Zhang, D.; Gao, F.; Nian, Y.; Zhou, Y.; Jiang, H.; Liu, H. Palladium-catalyzed picolinamide-directed coupling of C(sp $\left.{ }^{2}\right)-\mathrm{H}$ and $\mathrm{C}\left(\mathrm{sp}^{2}\right)-\mathrm{H}$ : A straightforward approach to quinolinone and pyridone scaffolds. Chem. Commun. 2015, 51, 7509-7511. [CrossRef]

28. Shan, G.; Yang, X.; Zong, Y.; Rao, Y. An efficient palladium-catalyzed C-H alkoxylation of unactivated methylene and methyl groups with cyclic hypervalent iodine $\left(\mathrm{I}^{3+}\right)$ oxidants. Angew. Chem. Int. Ed. 2013, 52, 13606-13610. [CrossRef]

29. Zong, Y.; Rao, Y. Developing Pd (II) catalyzed double $\mathrm{sp}^{3} \mathrm{C}-\mathrm{H}$ alkoxylation for synthesis of symmetric and unsymmetric acetals. Org. Lett. 2014, 16, 5278-5281. [CrossRef] [PubMed]

30. Sun, X.; Yao, X.; Zhang, C.; Rao, Y. Pd (II) catalyzed ortho C-H iodination of phenylcarbamates at room temperature using cyclic hypervalent iodine reagents. Chem. Commun. 2015, 51, 10014-10017. [CrossRef]

31. Yu, Q.-Y.; Zhong, H.-M.; Sun, W.-W.; Zhang, S.-J.; Cao, P.; Dong, X.-P.; Qin, H.-B.; Liu, J.-K.; Wu, B. Palladium-catalyzed C(sp ${ }^{3}$ )-H functionalization at the C3 position of L-pipecolinic acid derivatives. Asian J. Org. Chem. 2016, 5, 608-612. [CrossRef]

32. Yang, B.; Xu, X.-H.; Qing, F.-L. Synthesis of difluoroalkylated arenes by hydroaryldifluoromethylation of alkenes with $\alpha, \alpha-$ difluoroarylacetic acids under photoredox catalysis. Org. Lett. 2016, 18, 5956-5959. [CrossRef]

33. Nappi, M.; He, C.; Whitehurst, W.G.; Chappell, B.G.N.; Gaunt, M.J. Selective reductive elimination at alkyl palladium(IV) by dissociative ligand ionization: Catalytic $\mathrm{C}\left(\mathrm{sp}^{3}\right)-\mathrm{H}$ amination to azetidines. Angew. Chem. Int. Ed. 2018, 57, 3178-3182. [CrossRef] [PubMed]

34. Muraki, T.; Togo, H.; Yokoyama, M. Synthetic use of 1-(p-toluenesulfonyloxy)-1,2-benziodoxol-3(1H)-one: Iodination of aromatic rings. Synlett 1998, 3, 286-288. [CrossRef]

35. Muraki, T.; Togo, H.; Yokoyama, M. Reactivity and synthetic utility of 1-(arenesulfonyloxy)benziodoxolones. J. Org. Chem. 1999, 64, 2883-2889. [CrossRef]

36. Harper, M.J.; Emmett, E.J.; Bower, J.F.; Russell, C.A. Oxidative 1,2-difunctionalization of ethylene via gold-catalyzed oxyarylation. J. Am. Chem. Soc. 2017, 139, 12386-12389. [CrossRef]

37. Yoshimura, A.; Nguyen, K.C.; Klasen, S.C.; Postnikov, P.S.; Yusubov, M.S.; Saito, A.; Nemykin, V.N.; Zhdankin, V.V. Hypervalent iodine-catalyzed synthesis of 1,2,4-oxadiazoles from aldoximes and nitriles. Asian J. Org. Chem. 2016, 5, 1128-1133. [CrossRef]

38. Vinayak, B.; Ravindrakumar, P.V.; Ramana, D.V.; Chandrasekharam, M. Revisiting 1-chloro-1,2-benziodoxol-3-one: Efficient ortho-chlorination of aryls under aqueous conditions. New J. Chem. 2018, 42, 8953-8959. [CrossRef]

39. Parvathaneni, S.P.; Perumgani, P.C. Regioselective chlorination of aryl C-H bonds with hypervalent iodine(III) reagent 1-chloro1,2-benziodoxol-3-one. Asian J. Org. Chem. 2018, 7, 324-327. [CrossRef]

40. Jia, Y.; Chen, L.; Zhang, H.; Zheng, Y.; Jiang, Z.-X.; Yang, Z. Electrophilic chloro( $\omega$-alkoxy)lation of alkenes employing 1-chloro1,2-benziodoxol-3-one: Facile synthesis of $\beta$-chloroethers. Org. Biomol. Chem. 2018, 16, 7203-7213. [CrossRef]

41. Wang, M.; Zhang, Y.; Wang, T.; Wang, C.; Xue, D.; Xiao, J. Story of an age-old reagent: An electrophilic chlorination of arenes and heterocycles by 1-chloro-1,2-benziodoxol-3-one. Org. Lett. 2016, 18, 1976-1979. [CrossRef] [PubMed]

42. Egami, H.; Yoneda, T.; Uku, M.; Ide, T.; Kawato, Y.; Hamashima, Y. Difunctionalization of alkenes using 1-chloro-1,2-benziodoxol3-(1H)-one. J. Org. Chem. 2016, 81, 4020-4030. [CrossRef] [PubMed]

43. Xing, B.; Ni, C.; Hu, J. Hypervalent iodine(III)-catalyzed Balz-Schiemann fluorination under mild conditions. Angew. Chem. Int. Ed. 2018, 57, 9896-9900. [CrossRef]

44. Jiang, X.; Zheng, C.; Lei, L.; Lin, K.; Yu, C. Synthesis of 2-oxindoles from substituted indoles by hypervalent-iodine oxidation. Eur. J. Org. Chem. 2018, 2018, 1437-1442. [CrossRef]

45. Jiang, X.; Li, G.; Yu, C. A Diels-Alder reaction/oxa-Michael addition/acyloin rearrangement cascade on tropolonic substrates. Tetrahedron Lett. 2018, 59, 1506-1510. [CrossRef]

46. Vaillant, F.L.; Wodrich, M.D.; Waser, J. Room temperature decarboxylative cyanation of carboxylic acids using photoredox catalysis and cyanobenziodoxolones: A divergent mechanism compared to alkynylation. Chem. Sci. 2017, 8, 1790-1800. [CrossRef] [PubMed]

47. Li, X.; Qi, X.; Hou, C.; Chen, P.; Liu, G. Palladium(II)-catalyzed enantioselective azidation of unactivated alkenes. Angew. Chem. Int. Ed. 2020, 59, 17239-17244. [CrossRef]

48. Wang, Y.; Wang, N.; Zhao, J.; Sun, M.; You, H.; Fang, F.; Li, Z.-Q. Visible-light-promoted site-specific and diverse functionalization of a $\mathrm{C}\left(\mathrm{sp}^{3}\right)-\mathrm{C}\left(\mathrm{sp}^{3}\right)$ bond adjacent to an arene. ACS Catal. 2020, 10, 6603-6612. [CrossRef] 
49. Wang, Y.; Hu, X.; Morales-Rivera, C.A.; Li, G.-X.; Huang, X.; He, G.; Liu, P.; Chen, G. Epimerization of tertiary carbon centers via reversible radical cleavage of unactivated $\mathrm{C}\left(\mathrm{sp}^{3}\right)$-H bonds. J. Am. Chem. Soc. 2018, 140, 9678-9684. [CrossRef]

50. Shen, K.; Wang, Q. Copper-catalyzed alkene aminoazidation as a rapid entry to 1,2-diamines and installation of an azide reporter onto azaheterocycles. J. Am. Chem. Soc. 2017, 139, 13110-13116. [CrossRef] [PubMed]

51. Li, L.; Li, Z.-L.; Wang, F.-L.; Guo, Z.; Cheng, Y.-F.; Wang, N.; Dong, X.-W.; Fang, C.; Liu, J.; Hou, C.; et al. Radical aryl migration enables diversity-oriented synthesis of structurally diverse medium/macro- or bridged-rings. Nat. Commun. 2016, 7, 13852. [CrossRef]

52. Wang, Y.; Li, G.-X.; Yang, G.; He, G.; Chen, G. A visible-light-promoted radical reaction system for azidation and halogenation of tertiary aliphatic C-H bonds. Chem. Sci. 2016, 7, 2679-2683. [CrossRef] [PubMed]

53. Sharma, A.; Hartwig, J.F. Metal-catalyzed azidation of tertiary C-H bonds suitable for late-stage functionalization. Nature 2015, 600-604. [CrossRef]

54. Fumagalli, G.; Rabet, P.T.G.; Boyd, S.; Greaney, M.F. Three-component azidation of styrene-type double bonds: Light-switchable behavior of a copper photoredox catalyst. Angew. Chem. Int. Ed. 2015, 54, 11481-11484. [CrossRef] [PubMed]

55. Liu, T.; Zhu, J.; Sun, X.; Cheng, L.; Wu, L. I 2 /TBHP mediated divergent C(sp ${ }^{3}$ )-P cleavage of allenylphosphine oxides: Substituentcontrolled regioselectivity. Adv. Synth. Catal. 2019, 361, 3532-3537. [CrossRef]

56. Jiang, H.; He, Y.; Cheng, Y.; Yu, S. Radical alkynyltrifluoromethylation of alkenes initiated by an electron donor-acceptor complex. Org. Lett. 2017, 19, 1240-1243. [CrossRef]

57. Mangaonkar, S.R.; Kole, P.B.; Singh, F.V. Oxidation of organosulfides to organosulfones with trifluoromethyl 3-oxo-1 $\lambda^{3}$,2benziodoxole-1(3H)-carboxylate as an oxidant. Synlett 2018, 29, 199-202. [CrossRef]

58. Shen, K.; Wang, Q. Copper-catalyzed aminoalkynylation of alkenes with hypervalent iodine reagents. Chem. Sci. 2017, 8, 8265-8270. [CrossRef]

59. Mukherjee, S.; Garza-Sauchez, R.A.; Tlahuext-Aca, A.; Glorius, F. Alkynylation of $\mathrm{C}_{\mathrm{sp}}{ }^{2}(\mathrm{O})-\mathrm{H}$ bonds enabled by photoredoxmediated hydrogen-atom transfer. Angew. Chem. Int. Ed. 2017, 56, 14723-14726. [CrossRef]

60. Nicolai, S.; Waser, J. Pd(0)-catalyzed oxy- and aminoalkynylation of olefins for the synthesis of tetrahydrofurans and pyrrolidines. Org. Lett. 2011, 13, 6324-6327. [CrossRef]

61. Brand, J.P.; Waser, J. Direct alkynylation of thiophenes: Cooperative activation of TIPS-EBX with gold and Brønsted acids. Angew. Chem. Int. Ed. 2010, 49, 7304-7307. [CrossRef] [PubMed]

62. González, D.F.; Brand, J.P.; Waser, J. Asymmetric synthesis of 4-amino-4H-chromenes by organocatalytic oxa-Michael/aza-BaylisHillman tandem reactions. Chem. Eur. J. 2010, 16, 9457-9461.

63. Baker, G.P.; Mann, G.; Sheppard, N.; Tetlow, A.J. The structure of $o$-iodosobenzoic acid and of certain derivatives. J. Chem. Soc. 1965, 3721-3728. [CrossRef]

64. Kraszkiewicz, L.; Skulski, L. Optimized syntheses of iodylarenes from iodoarenes, with sodium periodate as the oxidant. Part II. Arkivoc 2003, 6, 120-125. [CrossRef]

65. Bosset, C.; Coffinier, R.; Peioto, P.A.; Assal, M.E.; Miqueu, K.; Sotiropoulos, M.; Pouységu, L.; Quideau, S. Asymmetric hydroxylative phenol dearomatization promoted by chiral binaphthylic and biphenylic iodanes. Angew. Chem. Int. Ed. 2014, 53, 9860-9864. [CrossRef] [PubMed]

66. Maity, A.; Hyun, S.-M.; Powers, D.C. Oxidase catalysis via aerobically generated hypervalent iodine intermediates. Nat. Chem. 2018, 10, 200-204. [CrossRef]

67. Chandrasekar, S.; Sekar, G. An efficient synthesis of iminoquinones by a chemoselective domino ortho-hydroxylation/oxidation/ imidation sequence of 2-aminoarylketones. Org. Biomol. Chem. 2016, 14, 3053-3060. [CrossRef]

68. Wirth, T. IBX-New reactions with an old reagent. Angew. Chem. Int. Ed. 2001, 40, 2812-2814. [CrossRef]

69. Ladziata, U.; Zhdankin, V.V. Hypervalent iodine(V) reagents in organic synthesis. Arkivoc 2006, 9, 26-58. [CrossRef]

70. Sharma, P.; Kaur, N.; Pareek, A.; Kishore, D. An insight in to general features of IBX (2-iodoxybenzoic acid). Sci. Revs. Chem. Commun. 2013, 3, 16-42.

71. Plumb, J.B.; Harper, D.J. IBX is reported to be a shock-sensitive explosive. Chem. Eng. News 1990, 68, 3.

72. Dess, D.B.; Martin, J.C.J. A useful 12-I-5 triacetoxyperiodinane (the Dess-Martin periodinane) for the selective oxidation of primary or secondary alcohols and a variety of related 12-I-5 species. J. Am. Chem. Soc. 1991, 113, 7277-7287. [CrossRef]

73. Boeckmann, R.K.; Shao, P.; Mullins, J.J. The Dess-Martin periodinane: 1,1,1-triacetoxy-1,1-dihydro-1,2-benziodoxolo-3(1H)-one. Org. Synth. 2000, 77, 141-152.

74. More, J.D.; Finney, N.S. A simple and advantageous protocol for the oxidation of alcohols with $o$-iodoxybenzoic acid (IBX). Org. Lett. 2002, 4, 3001-3003. [CrossRef]

75. Tiffner, M.; Stockhammer, L.; Schörgenhumer, J.; Röser, K.; Waser, M. Towards an asymmetric organocatalytic $\alpha$-azidation of $\beta$-ketoesters. Molecules 2018, 23, 1142. [CrossRef]

76. Bernini, R.; Barontini, M.; Spatafora, C. New lipophilic piceatannol derivatives exhibiting antioxidant activity prepared by aromatic hydroxylation with 2-iodoxybenzoic acid (IBX). Molecules 2009, 14, 4669-4681. [CrossRef]

77. Hussain, H.; Green, I.R.; Ahmed, I. Journey describing applications of oxone in synthetic chemistry. Chem. Rev. 2013, 113, 3329-3371. [CrossRef]

78. Thottumkara, A.P.; Bowsher, M.S.; Vinod, T.K. In situ generation of $o$-iodoxybenzoic acid (IBX) and the catalytic use of it in oxidation reactions in the presence of oxone as a co-oxidant. Org. Lett. 2005, 7, 2933-2936. [CrossRef] [PubMed] 
79. Uyanik, M.; Akakura, M.; Ishihara, K. 2-Iodoxybenzenesulfonic acid as an extremely active catalyst for the selective oxidation of alcohols to aldehydes, ketones, carboxylic acids, and enones with oxone. J. Am. Chem. Soc. 2009, 131, 251-262. [CrossRef]

80. Miura, T.; Nakashima, K.; Tada, N.; Itoh, A. An effective and catalytic oxidation using recyclable fluorous IBX. Chem. Commun. 2011, 47, 1875-1877. [CrossRef]

81. Moorthy, J.N.; Senapati, K.; Parida, K.N.; Jhulki, S.; Sooraj, K.; Nair, N.N. Twist does a twist to the reactivity: Stoichiometric and catalytic oxidations with twisted tetramethyl-IBX. J. Org. Chem. 2011, 76, 9593-9601. [CrossRef] [PubMed]

82. Ishihara, K.; Uyanik, M.; Crockett, R. 2-Iodoxy-5-methylbenzenesulfonic acid-catalyzed selective oxidation of 4-bromobenzyl alcohol to 4-bromobenzaldehyde or 4-bromobenzoic acid with oxone. Org. Synth. 2012, 89, 105-114.

83. Bikshapathi, R.; Prathima, P.S.; Rao, V.J. Hypervalent iodine catalysis for selective oxidation of Baylis-Hillman adducts via in situ generation of $o$-iodoxybenzoic acid (IBX) from 2-iodosobenzoic acid (IBA) in the presence of oxone. New J. Chem. 2016, 40, 10300-10304. [CrossRef]

84. Yakura, T.; Fujiwara, T.; Yamada, A.; Nambu, H. 2-Iodo- $N$-isopropyl-5-methoxybenzamide as a highly reactive and environmentally benign catalyst for alcohol oxidation. Beilstein J. Org. Chem. 2018, 14, 971-978. [CrossRef] [PubMed]

85. Mishra, A.K.; Moorthy, J.N. Mechanochemical catalytic oxidations in the solid state with in situ-generated modified IBX from 3,5-di-tert-butyl-2-iodobenzoic acid (DTB-IA)/Oxone. Org. Chem. Front. 2017, 4, 343-349. [CrossRef]

86. Jhulki, S.; Seth, S.; Mondal, M.; Moorthy, J.N. Facile organocatalytic domino oxidation of diols to lactones by in situ-generated TetMe-IBX. Tetrahedron 2014, 70, 2286-2293. [CrossRef]

87. Yakura, T.; Horiuchi, Y.; Nishimura, Y.; Yamada, A.; Nambu, H.; Fujiwara, T. Efficient oxidative cleavage of tetrahydrofuran2-methanols to $\gamma$-lactones by a 2-iodobenzamide catalyst in combination with Oxone ${ }^{\circledR}$. Adv. Synth. Catal. 2016, 358, 869-873. [CrossRef]

88. Yakura, T.; Yamauchi, Y.; Tian, Y.; Omoto, M. Catalytic hypervalent iodine oxidation of $p$-dialkoxybenzenes to $p$-quinones using 4-iodophenoxyacetic acid and Oxone ${ }^{\circledR}$. Chem. Pharm. Bull. 2008, 56, 1632-1634. [CrossRef] [PubMed]

89. Yakura, T.; Tian, Y.; Yamauchi, Y.; Omoto, M.; Konishi, T. Catalytic hypervalent iodine oxidation using 4-iodophenoxyacetic acid and Oxone ${ }^{\circledR}$ : Oxidation of $p$-alkoxyphenols to $p$-benzoquinones. Chem. Pharm. Bull. 2009, 57, 252-256. [CrossRef]

90. Zagulyaeva, A.A.; Banek, C.T.; Yusubov, M.S.; Zhdankin, V.V. Hofmann rearrangement of carboxamides mediated by hypervalent iodine species generated in situ from iodobenzene and oxone: Reaction scope and limitations. Org. Lett. 2010, 12, $4644-4647$. [CrossRef] [PubMed]

91. Yakura, T.; Omoto, M.; Yamauchi, Y.; Tian, Y.; Ozono, A. Hypervalent iodine oxidation of phenol derivatives using a catalytic amount of 4-iodophenoxyacetic acid and Oxone ${ }^{\circledR}$ as a co-oxidant. Tetrahedron 2010, 66, 5833-5840. [CrossRef]

92. Ren, J.; Lu, L.; Xu, J.; Yu, T.; Zeng, B.-B. Selective oxidation of 1-tetralones to 1,2-naphthoquinones with IBX and to 1,4naphthoquinones with Oxone ${ }^{\circledR}$ and 2-iodobenzoic acid. Synthesis 2015, 47, 2270-2280. [CrossRef]

93. Frigerio, M.; Santagostino, M.; Sputore, S. A user-friendly entry to 2-iodoxybenzoic acid (IBX). J. Org. Chem. 1999, 64, 4537-4538. [CrossRef]

94. China, H.; Tanihara, K.; Sasa, H.; Kikushima, K.; Dohi, T. Regiodivergent oxidation of alkoxyarenes by hypervalent iodine/oxone ${ }^{\circledR}$ system. Catal. Today 2020, 348, 2-8. [CrossRef]

95. Parida, K.N.; Moorthy, J.N. Synthesis of $o$-carboxyarylacrylic acids by room temperature oxidative cleavage of hydroxynaphthalenes and higher aromatics with Oxone. J. Org. Chem. 2015, 80, 8354-8360. [CrossRef]

96. Costello, J.P.; Ferreira, E.M. Regioselectivity influences in platinum-catalyzed intramolecular alkyne O-H and N-H additions. Org. Lett. 2019, 21, 9934-9939. [CrossRef]

97. Koguchi, S.; Mihoya, A.; Mimura, M. Alcohol oxidation via recyclable hydrophobic ionic liquid-supported IBX. Tetrahedron 2016, 72, 7633-7637. [CrossRef]

98. Boelke, A.; Kuczmera, T.J.; Caspers, L.D.; Lork, E.; Nachtsheim, B.J. Iodolopyrazolium salts: Synthesis, derivatizations, and applications. Org. Lett. 2020, 22, 7261-7266. [CrossRef]

99. Kalaj, M.; Mimeni, M.R.; Bentz, K.C.; Barcus, K.S.; Palomba, J.M.; Paesani, F.; Cohen, S.M. Halogen bonding in UiO-66 frameworks promotes superior chemical warfare agent simulant degradation. Chem. Commun. 2019, 55, 3481-3484. [CrossRef]

100. Sun, W.; Cama, L.D. Preparation of 6H-benzo[c]chromenes as estrogen receptor modulators. PCT Int. Appl. 2004, WO 2004073612, A2.

101. Caspers, L.D.; Spils, J.; Damrath, M.; Lork, E.; Nachtsheim, B.J. One-pot synthesis and conformational analysis of six-membered cyclic iodonium salts. J. Org. Chem. 2020, 85, 9161-9178. [CrossRef] [PubMed]

102. Miles, K.C.; Le, C.C.; Stambuli, J.P. Direct carbocyclizations of benzoic acids: Catalyst-controlled synthesis of cyclic ketones and the development of tandem aHH (acyl Heck-Heck) reactions. Chem. Eur. J. 2014, 20, 11336-11339. [CrossRef]

103. Hamley, P.; Pimm, A.; Tinker, A. Preparation of spiro[pyridoisoindolequinazoline] derivatives and analogs as nitric oxide synthase inhibitors. PCT Int. Appl. 1999, WO 9901455, A1.

104. Declas, N.; Vaillant, F.L.; Waser, J. Revisiting the Urech synthesis of hydantoins: Direct access to enantiopure 1,5-substituted hydantoins using cyanobenziodoxolone. Org. Lett. 2019, 21, 524-528. [CrossRef] [PubMed]

105. Bertho, S.; Rey-Rodriguez, R.; Colas, C.; Retailleau, P.; Gillaizeau, I. Regio- and stereoselective iron-catalyzed oxyazidation of enamides using a hypervalent iodine reagent. Chem. Eur. J. 2017, 23, 17674-17677. [CrossRef] [PubMed]

106. Lu, B.; Wu, J.; Yoshikai, N. Palladium-catalyzed condensation of $N$-aryl imines and alkynylbenziodoxolones to form multisubstituted furans. J. Am. Chem. Soc. 2014, 136, 11598-11601. [CrossRef] 
107. Chen, M.; Huang, Z.-T.; Zheng, Q.-Y. Organic base-promoted enantioselective electrophilic cyanation of $\beta$-keto esters by using chiral phase-transfer catalysts. Org. Biomol. Chem. 2015, 13, 8812-8816. [CrossRef]

108. Hari, D.P.; Waser, J. Enantioselective copper-catalyzed oxy-alkynylation of diazo compounds. J. Am. Chem. Soc. 2017, 139, 8420-8423. [CrossRef]

109. Mocci, F.; Uccheddu, G.; Frongia, A.; Cerioni, G. Solution structure of some $\lambda^{3}$ iodanes: An ${ }^{17}$ O NMR and DFT study. J. Org. Chem. 2007, 72, 4163-4168. [CrossRef]

110. Ito, E.; Fukushima, T.; Kawakami, T.; Murakami, K.; Itami, K. Catalytic dehydrogenative C-H imidation of arenes enabled by photo-generated hole donation to sulfonimide. Chem 2017, 2, 383-392. [CrossRef]

111. Jia, K.; Pan, Y.; Chen, Y. Selective carbonyl-C $\left(\mathrm{sp}^{3}\right)$ bond cleavage to construct ynamides, ynoates, and ynones by photoredox catalysis. Angew. Chem. Int. Ed. 2017, 56, 2478-2481. [CrossRef]

112. Iinuma, M.; Moriyama, K.; Togo, H. Oxidation of alcohols to aldehydes or ketones with 1-acetoxy-1,2-benziodoxole-3(1H)-one derivatives. Eur. J. Org. Chem. 2014, 2014, 772-780. [CrossRef] 